\title{
High-Resolution Confocal Fluorescence Imaging of Serine Hydrolase Activity in Cryosections - Application to Glioma Brain Unveils Activity Hotspots Originating from Tumor-Associated Neutrophils
}

Niina Aaltonen ${ }^{1 \dagger}$, Prosanta K. Singha ${ }^{1 \dagger}$, Hermina Jakupović ${ }^{1}$, Thomas Wirth ${ }^{2}$, Haritha Samaranayake², Sanna Pasonen-Seppänen ${ }^{1}$, Kirsi Rilla', Markku Varjosalo ${ }^{3}$, Laura E. Edgington-Mitchell ${ }^{4,5,6}$, Paulina Kasperkiewicz ${ }^{7}$, Marcin Drag ${ }^{7}$, Sara Kälvälä ${ }^{1}$, Eemeli Moisio ${ }^{1}$, Juha R. Savinainen ${ }^{1}$ and Jarmo T. Laitinen ${ }^{1 *}$ (1)

\begin{abstract}
Background: Serine hydrolases (SHs) are a functionally diverse family of enzymes playing pivotal roles in health and disease and have emerged as important therapeutic targets in many clinical conditions. Activity-based protein profiling (ABPP) using fluorophosphonate (FP) probes has been a powerful chemoproteomic approach in studies unveiling roles of SHs in various biological systems. ABPP utilizes cell/tissue proteomes and features the FPwarhead, linked to a fluorescent reporter for in-gel fluorescence imaging or a biotin tag for streptavidin enrichment and LC-MS/MS-based target identification. Existing ABPP approaches characterize global SH activity based on mobility in gel or MS-based target identification and cannot reveal the identity of the cell-type responsible for an individual SH activity originating from complex proteomes.

Results: Here, by using an activity probe with broad reactivity towards the SH family, we advance the ABPP methodology to glioma brain cryosections, enabling for the first time high-resolution confocal fluorescence imaging of global SH activity in the tumor microenvironment. Tumor-associated cell types were identified by extensive immunohistochemistry on activity probe-labeled sections. Tissue-ABPP indicated heightened SH activity in glioma vs. normal brain and unveiled activity hotspots originating from tumor-associated neutrophils (TANs), rather than tumor-associated macrophages (TAMs). Thorough optimization and validation was provided by parallel gelbased ABPP combined with LC-MS/MS-based target verification.

(Continued on next page)
\end{abstract}

\footnotetext{
* Correspondence: jarmo.laitinen@uef.fi

${ }^{\dagger}$ Niina Aaltonen and Prosanta K. Singha contributed equally to this work.

${ }^{1}$ Institute of Biomedicine, University of Eastern Finland (UEF), POB 1627,

Fl-70211 Kuopio, Finland

Full list of author information is available at the end of the article
}

(c) The Author(s). 2020 Open Access This article is licensed under a Creative Commons Attribution 4.0 International License, which permits use, sharing, adaptation, distribution and reproduction in any medium or format, as long as you give appropriate credit to the original author(s) and the source, provide a link to the Creative Commons licence, and indicate if changes were made. The images or other third party material in this article are included in the article's Creative Commons licence, unless indicated otherwise in a credit line to the material. If material is not included in the article's Creative Commons licence and your intended use is not permitted by statutory regulation or exceeds the permitted use, you will need to obtain permission directly from the copyright holder. To view a copy of this licence, visit http://creativecommons.org/licenses/by/4.0/ The Creative Commons Public Domain Dedication waiver (http://creativecommons.org/publicdomain/zero/1.0/) applies to the data made available in this article, unless otherwise stated in a credit line to the data. 


\begin{abstract}
(Continued from previous page)
Conclusions: Our study advances the ABPP methodology to tissue sections, enabling high-resolution confocal fluorescence imaging of global SH activity in anatomically preserved complex native cellular environment. To achieve global portrait of SH activity throughout the section, a probe with broad reactivity towards the SH family members was employed. As ABPP requires no a priori knowledge of the identity of the target, we envisage no imaginable reason why the presently described approach would not work for sections regardless of species and tissue source.
\end{abstract}

Keywords: Activity-based protein profiling (ABPP), Brain cryosection, Glioblastoma multiforme (GBM), Immunohistochemistry, Serine hydrolase activity, Neutrophil serine protease (NSP), TAMRA-FP probe, Tumorassociated neutrophils

\section{Background}

The serine hydrolases (SHs) form a diverse family of enzymes with a predicted number of $\sim 240$ in humans, falling into two subfamilies: the serine proteases $(\sim 125$ members $)$ and the metabolic SHs (mSHs $\sim 115$ members) [1,2]. The $\mathrm{mSHs}$ include small-molecule hydrolases, such as lipases, esterases and amidases and utilize a conserved serine nucleophile to hydrolyze amide, ester, and thioester bonds in different types of substrates including metabolites, lipids and peptides. Importantly, SHs have emerged as therapeutic targets in diseases such as cancer, obesity, diabetes, and neurological diseases [3].

The advent of chemoproteomic techniques some 20 years ago, activity-based protein profiling (ABPP) in particular, allowed for the first time proteome-wide profiling of SH activity in cells and tissue homogenates [4]. The prototype activity probe for $\mathrm{SHs}$ features the active sitetargeted warhead, typically a fluorophosphonate (FP), linked to a fluorescent reporter allowing in-gel imaging of $\mathrm{SH}$ activity in proteomes after SDS-PAGE separation. An advanced platform combining ABPP and multidimensional protein identification techniques (ABPP-MudPIT) was introduced to facilitate high-content functional proteomics discovery of potential new markers of human diseases [5].

In the comparative mode, ABPP enables comparison of $\mathrm{SH}$ activity pattern between different proteomes, e.g. aggressive vs. non-aggressive cancer cells [6]. Indeed, such studies have highlighted previously unrecognized importance of $\mathrm{SH}$ family members as metabolic nodes orchestrating the availability of lipids involved in oncogenic signaling.

In the competitive mode, ABPP has proven its power in the discovery and selectivity testing of novel inhibitors targeting individual SHs [7]. In this approach, the proteome is first treated with the inhibitor (commonly a serinenucleophile targeting covalent inhibitor), binding of the inhibitor masks the active site, preventing subsequent labeling with the activity probe. EnPlex, an advanced highthroughput platform based on ABPP principles has been introduced as a feasible approach for $\mathrm{SH}$ superfamily-wide selectivity profiling that could be incorporated into the early stage of drug discovery [8].

ABPP can also serve a powerful platform for massspectrometry (MS) -based target identification from complex proteomes. In this case, the FP-warhead is linked to a biotin tag, enabling streptavidin enrichment and subsequent LC/MS-MS-based target identification.

Current ABPP approaches characterize global $\mathrm{SH}$ activity based on mobility in gel or MS-based target identification from homogenates and cannot reveal the identity of the cell-types responsible for an individual $\mathrm{SH}$ activity originating from complex proteomes, suggesting that the full potential of this technology may not have yet been harnessed. To date, quenched activity probe-based live cell fluorescence imaging has been described for a handful of proteases, such as cysteine cathepsins [9]. The quenched fluorescent probes were applied for labeling of active proteases in fresh-frozen cancer tissues [10], for macrophage detection in atherosclerotic plaques [11], and as a diagnostic tool to image skin tumor margins [12]. Recently, a small-molecule chemical toolbox was constructed for parallel imaging of human neutrophil serine proteases (NSPs). The toolbox comprised activity-probes with different fluorophores showing minimal wavelength overlap and highly selective natural and unnatural amino acid recognition sequences tailored for the four individual NSPs [13]. This elegant approach enabled for the first-time simultaneous imaging of the four NSPs in living neutrophils by fluorescence microscopy.

We reasoned that ABPP-based imaging of SH activity should be feasible at high resolution in complex native proteomes such as brain tissue while preserving the anatomical details of this delicate organ. Brain cryosections serve as fascinating premise to directly explore this issue as functional responses such as receptor-stimulated $\mathrm{G}$ protein activity and $\mathrm{mSH}$ regulated endocannabinoid tone can be readily monitored in brain cryosections without compromising the anatomical integrity $[14,15]$. 
Here, by using an activity probe with broad reactivity towards the $\mathrm{SH}$ family, we advance the ABPP methodology to glioma brain cryosections, enabling high-resolution confocal fluorescence imaging of global $\mathrm{SH}$ activity in glioma brain with well-preserved cyto-architecture. We unveil heightened $\mathrm{SH}$ activity in the tumor, as compared to healthy brain. Heterogeneous distribution pattern of $\mathrm{SH}$ activity within the tumor microenvironment was explored in detail by identification of the glioma-associated cell types using immunohistochemical markers. Crossvalidation was provided by classical gel-based ABPP using homogenates of healthy brain and glioma, as well as by LC/MS-MS-based target identification. These studied revealed quite unexpectedly that $\mathrm{SH}$ activity hotspots in glioma originate from tumor-associated neutrophils (TANs), rather than tumor-associated macrophages (TAMs). We anticipate that tissue-ABPP should enable a wide range of applications for high-resolution confocal imaging of global $\mathrm{SH}$ activity in practically any type of tissue and species, opening new avenues for cellular and subcellular localization of $\mathrm{SH}$ activity in complex proteomes with preserved anatomy.

\section{Results}

The principal motivation for this work was to extend the utility of ABPP towards applications enabling highresolution imaging of global $\mathrm{SH}$ activity in cryosections of complex proteome, namely glioma brain, while preserving the delicate cyto-architecture of the tumor microenvironment. We chose to use a promiscuous activity probe for these studies in order to portrait the global $\mathrm{SH}$ activity profile in glioma brain sections. The tissue- and gel-based ABPP approaches that were utilized in this study are illustrated in Figure S1.

\section{The Glioma Model}

Glioblastoma multiforme (GBM) is the most malignant and most frequent brain tumor accounting for more than $65 \%$ of all cases [16]. As the name implies, GBMs have a wide spectrum of histological morphologies ranging from small-cell type to very pleomorphic giant-cell forms with poor differentiation and gliosarcomas [16, 17]. We used the rat syngeneic BT4C gliosarcoma model [18] in which the glioma grows in a sarcomatous pattern and is typically composed of a pleomorphic population of tumor cells (Figure S2). We imaged tumors in vivo 22-24 days after implantation using MRI (Figure S3) and animals were sacrificed 5-11 days later. Brain tissue was excised from saline-perfused animals and used for cryosectioning (tissue-ABPP) or preparation of homogenates that were used in cross-validation experiments by gelABPP. Animals of both sexes were used.

\section{Optimization and Validation of ABPP Protocol for Brain Cryosections}

As our ultimate goal was to achieve high-resolution imaging of $\mathrm{SH}$ activity in cryosections without significantly compromising anatomical integrity, we considered fixation necessary to preserve delicate cyto-architecture. On the other hand, fixation should be sufficiently mild in order to preserve enzymatic activity, a prerequisite for the probe to covalently label catalytically competent SHs. We found that in contrast to acetone or methanol, fixation with paraformaldehyde preserved tissue integrity and inhibitor sensitivity of TAMRA-FP labeling (Figure S4).

We chose $0.5 \mu \mathrm{M}$ probe concentration as a compromise between signal intensity and cost-affordable probe amount (Figure S5). Concerning assay buffer, we found that for applications where maximal TAMRA-FP signal is the desired final readout, Tris or phosphate buffer ( $\mathrm{pH}$ 7.4) without BSA supplementation is optimal. However, for applications where TAMRA-FP labeling step is followed by immunohistochemistry, BSA is included to block tissue prior to antibody addition (Figure S6).

\section{Distribution and Overall Characteristics of TAMRA-FP Signal in Glioma and Control Brain}

As shown in Fig. 1, relatively intense and heterogeneously distributed TAMRA-FP labeling was evident over the glioma. In general, TAMRA-FP fluorescence was more intense over the glioma as compared to most regions of the healthy brain. Nuclear DAPI staining indicated dense cell population in glioma as compared to most regions of the healthy brain. This likely partly accounts for the heightened TAMRA-FP signal in glioma. Intense TAMRA-FP labeling was evident also in celldense structures of the healthy brain, such as hippocampal pyramidal cell layer and granular layer of dentate gyrus (Fig. 1a) and cerebellar Purkinje cell layer (Figure S6). On the other hand, white matter tracts (Fig. 1a) showed low TAMRA-FP labeling. Collectively these findings indicate that under the conditions employed, the method is sufficiently sensitive to allow imaging of TAMRA-FP labeling not only over the tumor, but also in various regions of the healthy brain.

Building on tissue-ABPP images of gliomas from different animals, a common pattern of TAMRA-FP labeling emerged and the following classification will be used to facilitate signal interpretation in forthcoming immunohistochemistry. A common TAMRA-FP labeling pattern with widest distribution over the glioma was characterized by non-nuclear intense fluorescence originating from evenly distributed individual cells (Fig. 1b, Video S1). In what follows, we define these cells "TAMRA-FP hotspots". However, the most intense TAMRA-FP labeling pattern was non-nuclear and originated from cell clusters with variable size (Fig. 1c, Video S1). We define this labeling pattern 

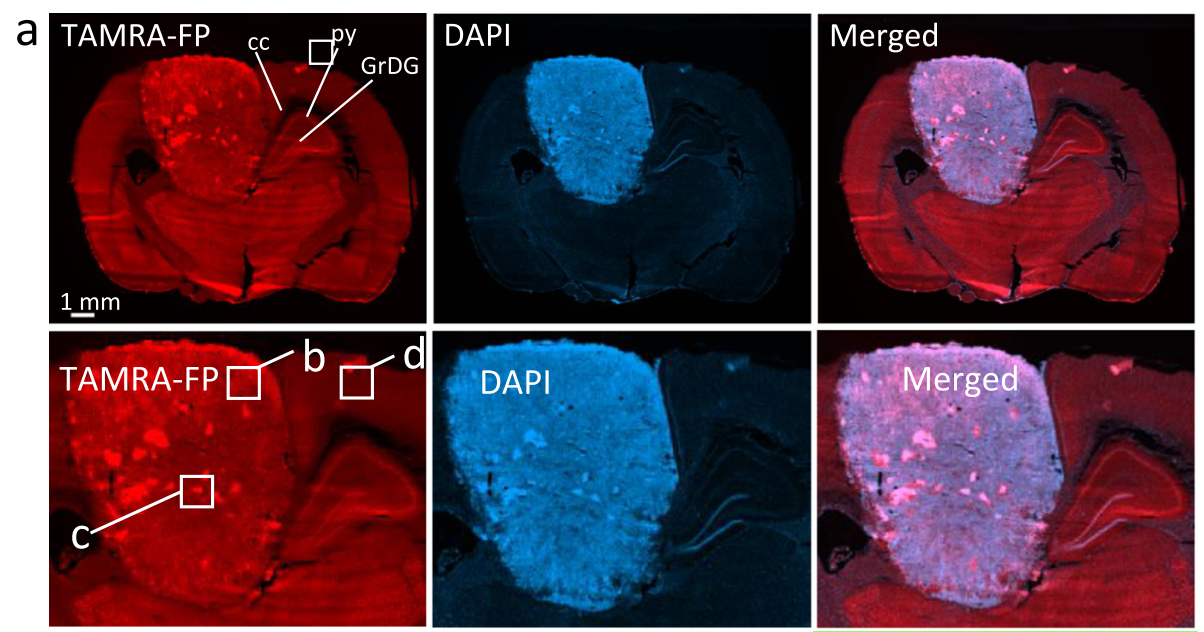

b
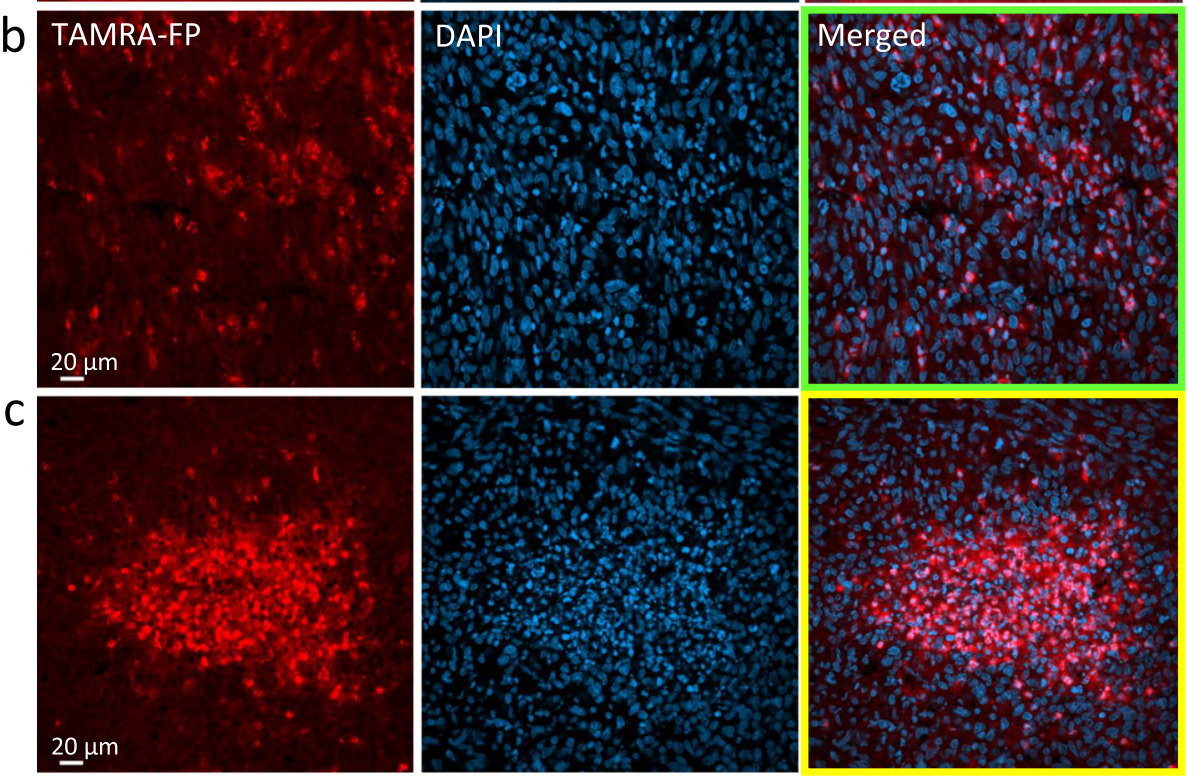

d
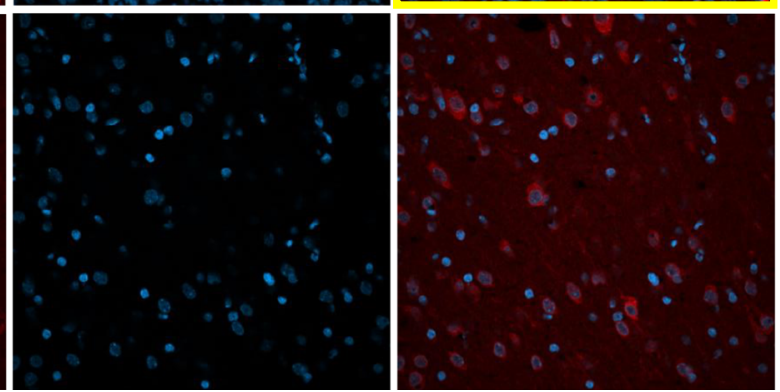

Fig. 1 (See legend on next page.)

"TAMRA-FP hotspot clusters". In healthy cortex shown for comparison, TAMRA-FP fluorescence was less intense and mainly localized to cytosol and plasma membrane (Fig. 1d).

\section{Probe Labeling of Tumor and Healthy Brain SHs is}

Differentially Sensitive to SH Inhibitors

To assure that TAMRA-FP specifically reports $\mathrm{SH}$ activity under the conditions of tissue-ABPP, we used the competitive approach testing a panel of serinenucleophile targeting broad-spectrum inhibitors, containing either FP [DeBi-FP (desthiobiotin-FP), MAFP (methyl arachidonyl fluorophosphonate), IDFP (isopropyl dodecylfluorophosphonate)] or sulfonylfluoride [AEBSF (aminoethylbenzenesulfonyl fluoride), PMSF (phenylmethylsulfonyl fluoride)] as the warhead. Inhibitors were purposely used at maximally effective 
(See figure on previous page.)

Fig. 1 Distribution and overall characteristics of TAMRA-FP signal in glioma and control brain. Note relatively intense and heterogeneously distributed TAMRA-FP fluorescence over the glioma as compared to most regions of the healthy brain. Nuclear DAPI staining indicates dense cell population in glioma as compared to most regions of the healthy brain. Note relatively intense TAMRA-FP and DAPI signal also in cell-dense hippocampal pyramidal cell layer (py) and granular layer of dentate gyrus (GrDG) as well as relatively weak TAMRA-FP signal in white matter tracts of corpus callosum (cc) (a), suggesting that the method offers sufficient sensitivity to enable imaging of TAMRA-FP fluorescence not only in the tumor, but also in various regions of the healthy brain. Building on tissue-ABPP images of gliomas from different animals, a common pattern of TAMRA-FP labeling was established: TAMRA-FP hotspots (b), characterized by intense and widely-distributed non-nuclear TAMRA-FP signal over the glioma, originating from evenly distributed individual cells. We define the second pattern as TAMRA-FP hotspot clusters (c), characterized by intense non-nuclear TAMRA-FP signal originating from cell clusters. In healthy cortical region shown for comparison (d), TAMRA-FP signal is less intense and localizes mainly to cytosol and plasma membrane. The section illustrated here for TAMRA-FP and DAPI staining was further immunostained for the phagocyte marker CD11b/c and is presented again (Figure S18). 3D-animation of merged TAMRA-FP-DAPI fluorescence throughout the section thickness in TAMRA-FP hotspots (green lining) and TAMRA-FP hotspot clusters (yellow lining) is shown in

Supplementary Video 1

concentrations to ensure comprehensive blockade of $\mathrm{SH}$ activity prior to TAMRA-FP labeling. As evident from Fig. 2, desthiobiotin-FP sharing the warhead-linker moiety with TAMRA-FP, effectively inhibited TAMRA-FP binding throughout the brain section, although weak residual labeling was evident as sparse spots over the glioma. Further, treatment with PMSF and IDFP efficiently prevented probe labeling in most brain regions, although some residual signal persisted over the tumor. Intriguingly, while the $\mathrm{mSH}$-inhibitor MAFP efficiently blocked probe binding in most regions of the healthy brain, it was less effective in preventing TAMRA-FP labeling of the tumor, indicating that while MAFPsensitive $\mathrm{SH}$ activity predominated in normal brain, $\mathrm{SH}$ activity originating from glioma was rather insensitive to this inhibitor. We considered this as a notable finding, as MAFP is known to act as a pan-SH inhibitor potently targeting the vast majority of $\mathrm{mSHs}$ [8]. When applied to rat brain cryosections in a comparable setting, our previous study indicated that MAFP $(10 \mu \mathrm{M})$ was able to comprehensively block mSH-mediated hydrolysis of the major endocannabinoid 2-arachidonoylglycerol [14].

\section{Comparative Gel-Based ABPP of SH Activity in Homogenates of Glioma and Control Brain}

We cross-validated the tissue-ABPP findings in gelbased ABPP using homogenates of glioma and control brain. In addition, we used rat cerebellar membranes to facilitate comparison, as previous gel-based ABPP has substantially relied on $\mathrm{SH}$ profiling in rodent brain membrane proteomes [14, 19-27]. Building on such studies from this and other laboratories [14, 19-27], we could identify many of the SH bands based on inhibitor sensitivity and mobility pattern in SDS-PAGE. These studies revealed that while many of the SH bands were common to the three proteomes (Fig. 3), brain-resident SHs, including monoacylglycerol lipase (MAGL 35 $\mathrm{kDa})$ and KIAA1363 $(\sim 50 \mathrm{kDa})$ showed variable activity in glioma. Both were proposed to play protumorigenic role in various cancers [6]. While comparable activity was evident for KIAA1363, MAGL showed low-to-nondetectable activity in glioma, suggesting that it might not play a major role in this glioma model. In addition, two distinct $\mathrm{mSHs}$, namely lysophospholipases $\mathrm{A} 1$ and A2 (LYPLA1/2 $\sim 25 \mathrm{kDa}$ ) showed prominent, yet comparable activity in control brain and glioma. LYPLA1/2 are depalmitoylases regulating membrane-association and oncogenic signaling of Ras, and are inhibited by palmostatin B ([28] and references therein).

Of note, the glioma possessed two prominent $\mathrm{SH}$ bands migrating at $\sim 25$ and $\sim 30 \mathrm{kDa}$ (Fig. 3). It is noteworthy that the respective $\mathrm{SH}$ bands showed low-tonon-detectable activity in control brain. We verified that the profile differences were present in homogenates from seven glioma rats (Figure S7). Inhibitor profiling revealed that the $\sim 30 \mathrm{kDa}$ band was sensitive to all tested inhibitors whereas the $\sim 25 \mathrm{kDa}$ band was sensitive to PMSF and deshiobiotin-FP, being resistant to other inhibitors, including MAFP. Collectively, the tissue and gel-based ABPP experiments suggest that MAFPresistant TAMRA-FP hotspots in the tumor likely reflects activity of the dominant $\sim 25 \mathrm{kDa}$ band, while the bulk of MAFP-sensitive SH activity likely represent activity of the $\sim 30 \mathrm{kDa}$ prominent $\mathrm{SH}$ band. Noteworthy, in line with the outcome with glioma sections, PMSF efficiently prevented probe labeling of glioma homogenate in gel-ABPP (Fig. 3). The gel-ABPP findings not only cross-validate findings from tissue-ABPP but additionally indicate that the $\mathrm{SH}$ activity profile of glioma is distinct from that of normal brain and must therefore reflect activity originating from cell types not normally residing in brain.

\section{Tissue-ABPP Combined with Immunohistochemistry Enables Subcellular Localization of SH Activity within the Tumor Microenvironment}

The glioma is a highly heterogeneous cancer and consists of several cell types, including tumor cells, endothelial cells from angiogenic vessels, neurons, scar-forming astroglia, as well as infiltrating monocytes/macrophages 

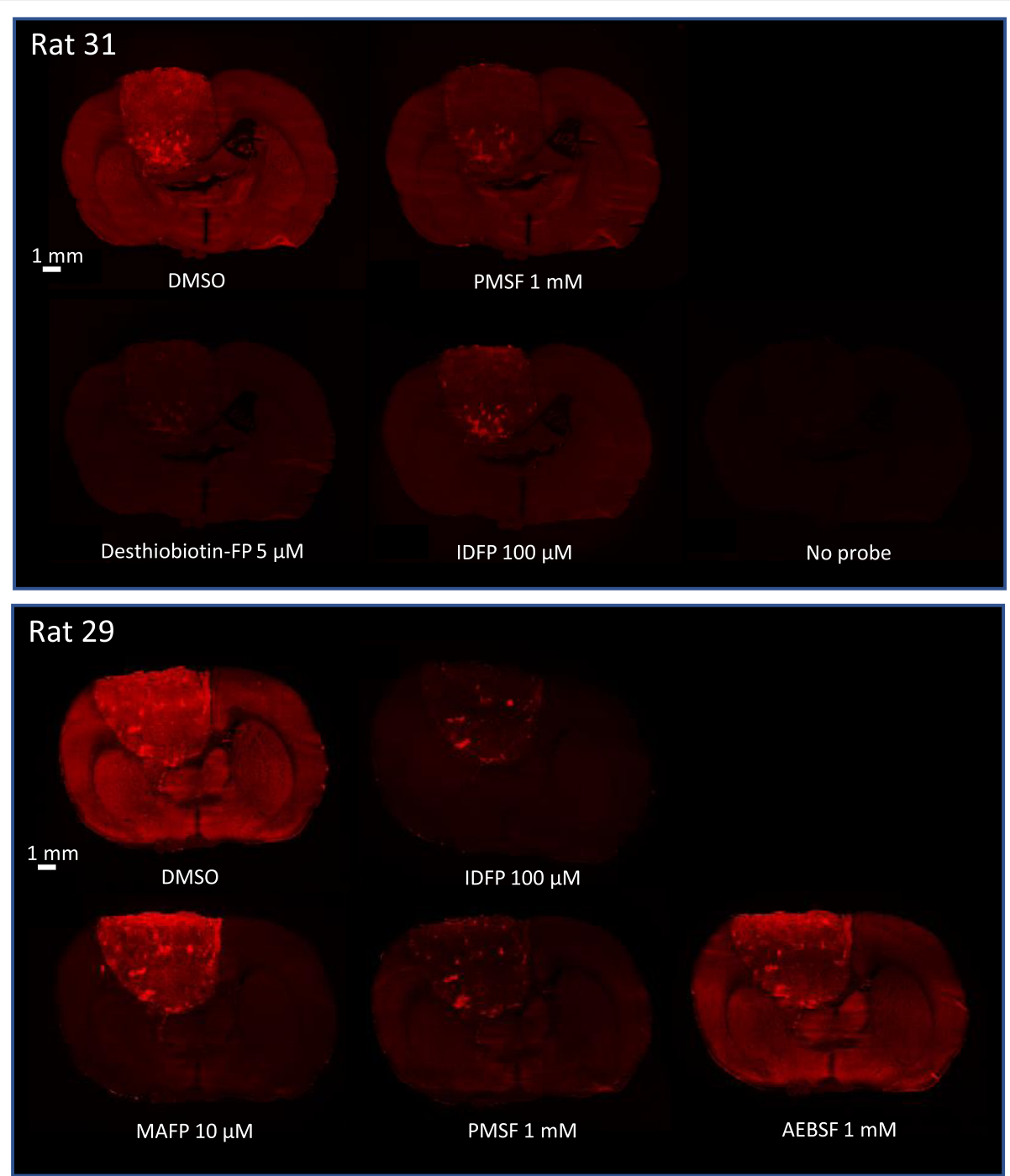

Fig. 2 Competitive tissue-ABPP demonstrating that TAMRA-FP reports SH activity in glioma brain sections. Sections were pretreated for $1 \mathrm{~h}$ with the indicated concentrations of various serine-nucleophile targeting inhibitors and processed for TAMRA-FP labeling and confocal fluorescence imaging as detailed in Materials and Methods. Sections from two individual rats bearing the tumor were used and images from two independent experiments are shown as separate panels with DMSO control included in both experiments. Desthiobiotin-FP $(5 \mu M)$ efficiently inhibits TAMRAFP labeling throughout the brain section. However, a weak residual signal persists as spots over the glioma tissue. Note absence of fluorescence in the section processed without TAMRA-FP, indicating no detectable autofluorescence in the Cy3-window used for TAMRA-FP imaging. Note that PMSF $(1 \mathrm{mM})$, in contrast to AEBSF $(1 \mathrm{mM})$, and IDFP $(100 \mu \mathrm{M})$ both efficiently inhibit TAMRA-FP labeling throughout the brain sections, yet leaving some residual activity over the tumor. Note in particular that MAFP $(10 \mu \mathrm{M})$ efficiently inhibits probe labeling throughout the healthy brain regions but only modestly inhibited labeling of TAMRA-FP hotspots in the tumor. However, partial MAFP-sensitivity of TAMRA-FP signal was evident in tumors regions showing moderate probe fluorescence. The scale bars represent $1 \mathrm{~mm}$. Images were adjusted for brightness and contrast

that are recruited from the periphery [29-31]. We approached this cellular complexity by immunohistochemistry using various cell-type markers that were applied on TAMRA-FP labeled sections.

To visualize tumor cells, sections were stained for Ki67, a nuclear marker of proliferating cell populations. Proliferating scattered cell populations were detected in glioma but as expected, were absent from healthy brain (Figure S8). Ki67-positive cells showed poor co- localization with TAMRA-FP hotspots or TAMRA-FP hotspot clusters.

GFAP-positive astrocytes appeared as a dense cell population encircling the tumor (Figure S9). Although scattered GFAP-positive cells were detected within the tumor, the bulk of tumor was devoid of astrocytes, in line with previous findings with this model [29]. The sparse presence of astrocytes is consistent with the gliosarcoma character of this model [18]. Although 


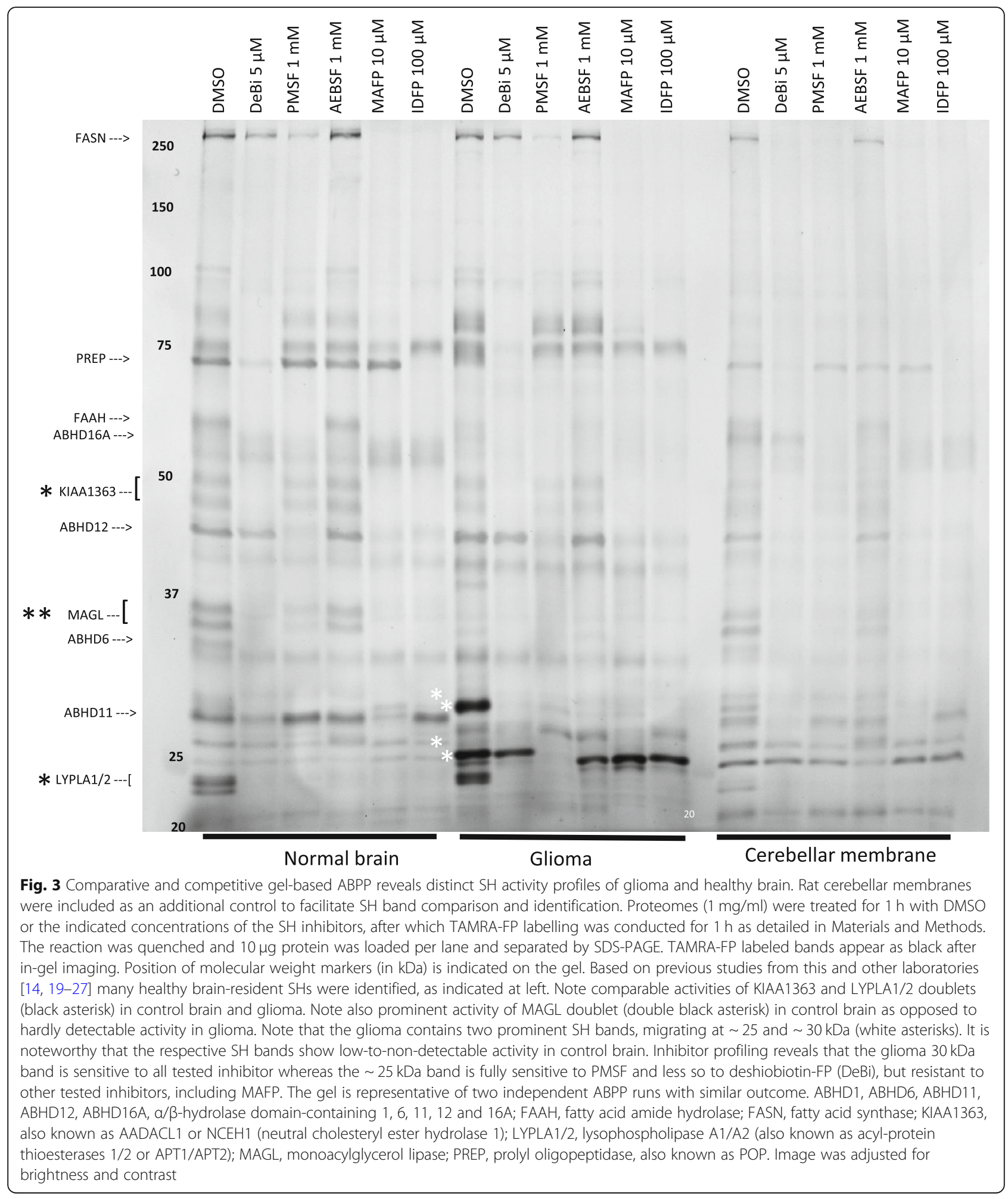

astrocytes showed moderate $\mathrm{SH}$ activity, TAMRA-FP hotspots or TAMRA-FP hotspot clusters showed poor co-localization with GFAP-positive cells. Throughout the healthy brain, cells with star-shaped morphology (i.e. astrocytes) were visible.
Tumor vasculature, visualized using the endothelial markers von Willebrand factor and CD34, revealed relatively low TAMRA-FP signal originating from endothelial cells (Figure S10). However, TAMRA-FP hotspot clusters resided often in the vicinity of the vessels. This 
glioma model shows leaky blood vessels with compromised blood-brain-barrier (BBB) function, especially at the tumor edge (Figure S1). Sections were stained for the redox-sensitive marker heme oxygenase-1 (HO-1) to visualize areas prone to redox stress (Figure S11). A heterogeneous pattern of $\mathrm{HO}-1$ staining was evident in the tumor with no detectable signal in control brain. However, although HO-1 positive cells localized to regions of intense SH activity, neither TAMRA-FP hotspots nor TAMRA-FP hotspot clusters stained strongly for HO-1.

In the absence of truly selective microglial marker, we stained sections for Iba1 (Figure S12), being aware of that this marker also labels activated monocytes and macrophages. We observed heterogeneous presence of Iba1-positive cells throughout the tumor with intense staining in tumor margins, and noted partial overlap of Iba1 staining with TAMRA-FP hotspots. However, no Iba1-positive cells were evident in tumor regions of TAMRA-FP hotspot clusters. As expected, Iba1-positive cells with characteristic microglial morphology were detected in the healthy brain (Figure S12).

\section{SH Activity in Relation to Hyaluronan (HA) - CD44 Axis and Markers of Tumor Stiffness}

Stromal HA accumulation is considered as a protumorigenic factor in several solid tumors [32, 33]. Extensive modeling of the extracellular matrix associated with biomechanical changes, often called tumor "stiffness", is a hallmark of "cancerized" fibrotic stroma [32]. The HA receptor CD44 promotes cancer cell motility, tumor growth, angiogenesis as well as resistance to chemo- and radiotherapy, and is overexpressed in various tumors, including GBM [34]. We clarified whether TAMRA-FP hotspots localize to tumor regions undergoing matrix remodeling using the stiffness markers pMLC2 and tenascin $\mathrm{C}$ along with HA and CD44. HA was detected in the extracellular matrix throughout the brain with more intense staining in glioma, especially the glioma edges (Figure S13). HA was abundant in areas of TAMRA-FP hotspots. In contrast, HA was sparse in regions of TAMRA-FP hotspot clusters, whereas these clusters were surrounded by HA-enriched tissue. Like HA, CD44 was enriched in the glioma with low expression in normal brain (Figure S14). Like HA, CD44 was abundant in glioma regions of TAMRA-FP hotspots but was undetectable in regions of TAMRA-FP hotspot clusters, yet these clusters were surrounded by CD44-positive cells. pMLC2 showed intense staining over the glioma but was absent from healthy brain (Figure S15). Interestingly, the stiffness marker frequently co-localized with TAMRAFP hotspots. In contrast, pMLC2-positive cells were rare in the central part of TAMRA-FP hotspot clusters whereas TAMRA-FP-positive cells at the edge of these clusters expressed pMLC2 and were also surrounded by
pMLC2-positive cells. The outcome with tenascin C (Figure S16) was similar to what was observed with pMLC2.

\section{SH Activity in Relation to Cells of Myeloid and Lymphoid Origin}

Bone-marrow-derived cell populations are abundant in tumors, including GBM [30, 31]. In human and rodent gliomas, tumor-associated macrophages (TAMs) are the major class of tumor-promoting immune cells and were also previously detected in this model [29]. We pursued to identify TAMs and other bone-marrow-derived cells in glioma. Interestingly, both TAMRA-FP hotspots and hot spot clusters expressed the hematopoietic marker CD45 (Figure S17) or the phagocyte marker CD11b/c (Figure S18). Although macrophages were abundant in glioma regions showing prominent $\mathrm{SH}$ activity, neither TAMRA-FP hotspots nor TAMRA-FP hotspot clusters originated from cells stained for the macrophage markers CD68 (Figure S19), CD163 (Figure S20) or CD169 (Figure S21). Lymphoid cells were present in glioma regions showing prominent $\mathrm{SH}$ activity and TAMRA-FP hotspots partially overlapped with CD4and CD8-positive cells, whereas TAMRA-FP hotspot clusters sparsely expressed the T-cell markers (Figures S22 and S23).

\section{Tumor-Associated Mast Cells Partly Account for the High SH Activity}

We found low expression of the mast cell marker Fc\&RIy within TAMRA-FP hotspots whereas FceRI $\gamma$-positive cells were enriched in TAMRA-FP hotspot clusters, showing marked co-localization with TAMRA-FP (Figure S24). Besides mast cells, FceRIy labels eosinophils, basophils and monocytes, so this marker alone cannot verify the presence of mast cells. Mast cell-specific serine proteases include tryptases and chymases. We stained the sections for chymase using CMA1 antibody. Faint expression of CMA1 was evident in glioma where CMA1-positive cells co-localized with TAMRA-FP hotspots, whereas CMA1-positive cells were scarce in TAMRA-FP hotspot clusters (Figure S25). Unfortunately, we found no additional antibodies that qualified for further verification of mast cell presence. Further, attempts to detect mast cells by enzymo-histochemical staining of serine protease activity using peptide substrates tailored for human tryptase and chymase [35] were unsuccessful (data not shown).

Tumor-Associated Neutrophils (TANs) Largely Account for Tumor SH Activity Hotspots

Neutrophils encompass four serine proteases (NSPs), namely neutrophil elastase (NE), cathepsin G (CTSG), proteinase 3 (PR3), and neutrophil proteinase 4 (NSP4, 
also known as PRSS57) [13, 36]. We stained glioma sections for the neutrophil marker myeloperoxidase (MPO) and found that MPO-positive cells localized to TAMRAFP hotspots (Fig. 4). Further, MPO-positive cells were abundant in TAMRA-FP hotspot clusters (Fig. 4). The outcome was essentially the same in sections stained for the NSP elastase (Fig. 5).

Noteworthy, high-resolution fluorescence imaging revealed that TAMRA-FP hotspots had the morphological characteristics of neutrophil-type multi-nucleated cells (Fig. 6). Importantly, TAMRA-FP labeling of these cells was sensitive to the SH inhibitors PMSF and DeBi-FP, indicating that TAMRA-FP fluorescence in the glioma hotspots and hotspot clusters indeed represents $\mathrm{SH}$ activity.

In sham-operated animals, TAMRA-FP hotspots with TAN-like morphology were rare at the site of injection (Figure S26), indicating that the surgical operation per se did not cause TAN accumulation. Collectively these findings indicated that TANs represent the principal cell-type accounting for TAMRA-FP hotspots and hotspot clusters.

\section{ABPP of Glioma Proteome Using Novel Activity Probes Designed for Human Serine Proteases}

In further efforts to characterize glioma serine protease activity, we utilized newly introduced activity probes with preference for trypsin and elastase [9] using both gel- and tissue-based ABPP approaches. These probes bear a diphenylphosphonate (DPP) warhead, which targets serine proteases more specifically than the FP warhead which broadly targets the mSH family [37]. Besides glioma, we profiled tumor cells and bone-marrowderived mononuclear cells (rBM). Interestingly, the elastase-preferring probe V-DPP detected 3-4 proteins $(\sim 25 \mathrm{kDa})$ in $\mathrm{rBM}$ and weakly two bands of similar size in glioma (Figure S27). Probe binding was sensitive to $\mathrm{SH}$ inhibitors, indicating that V-DPP specifically reported elastase-type serine protease activity. The trypsinpreferring probe PK-DPP gave no detectable signal in gel-ABPP (Figure S27). When tested in tissue-ABPP in place of TAMRA-FP, probe binding was hardly visible and neither probe labeled inhibitor-sensitive targets in glioma (Figure S28).

We evaluated also novel activity probes bearing the DPP warhead that were tailored to report individually activities of the four human NSPs [13]. Unfortunately, the NSP-probes did not recognize any TAMRA-FP sensitive bands in gel-ABPP of rat proteomes (Figure S29). When tested in tissue-ABPP in place of TAMRA-FP, the NSP-probes readily bound to sections, yet in a nonspecific manner, as probe binding was not prevented by prior treatment with TAMRA-FP (Figure S29).
As a final step, we used gel-ABPP to assess inhibitor sensitivity of TAMRA-FP labeled proteins in neutrophils and rBMs (Figure S30). Only few prominent bands migrating at $\sim 25-30 \mathrm{kDa}$ were present in these proteomes and TAMRA-FP labeling of these bands was inhibited by PMSF, desthiobiotin-FP and AEBSF, but was largely resistant to MAFP and IDFP, consistent with the pharmacology of the $\sim 25 \mathrm{kDa}$ glioma band. Compound 22, designed to potently target human CTSG [38], was inactive in the rat proteomes whereas another CTSG inhibitor (CTSG-I) blocked TAMRA-FP labeling of the $25 \mathrm{kDa}$ band in both samples. In tissue-ABPP, the inhibitor did not prevent TAMRA-FP labeling of hotspots or hotspot clusters (Figure S30).

\section{LC-MS/MS Analysis of the Glioma SH Bands Migrating at $\sim 25-30 \mathrm{kDa}$}

Gel-pieces encompassing the prominent $\mathrm{SH}$ bands in glioma, rBMs and tumor cells were cut after in-gel fluorescence scanning and subjected to LC-MS/MS-based target identification. To facilitate SDS-PAGE separation of proteins with similar size, proteomes were deglycosylated prior to TAMRA-FP labeling. The complete LCMS/MS data with all identified proteins is available as Supplementary File 1. Interestingly, neutrophil serine proteases CTSG (Ctsg), NE (Elane) and PR3 (Prtn3) were among the SHs present in the $\sim 25 \mathrm{kDa}$ gel-pieces of glioma and bone marrow samples (Fig. 7), in strong support for findings from tissue-ABPP. Interestingly, the $\sim 25-30 \mathrm{kDa}$ gel-pieces encompassed also two MAFPsensitive SHs [8], namely PAF-acetylhydrolases $1 \mathrm{~b} 2$ and 1b3 (PAFAH1b3 and PAFAH1b2). Collectively, our experiments indicate that the MAFP-resistant TAMRA-FP signal in the tumor is principally due to neutrophil serine proteases migrating in the $\sim 25 \mathrm{kDa}$ band, while the bulk of MAFP-sensitive activity in glioma is likely due to PAFAH1b3 and PAFAH1b2.

\section{Discussion}

Our study widens the applicability of the chemoproteomic technology to tissue sections, enabling for the first time high-resolution confocal fluorescence imaging of family-wide $\mathrm{SH}$ activity in anatomically preserved native cellular environment. Thorough optimization and validation was provided by parallel gel-based ABPP, extensive immunohistochemical mapping of TAMRA-FP signal origin, as well as LC-MS/MS-based target verification. Collectively all the evidence demonstrates convincingly that tissue-ABPP faithfully reports $\mathrm{SH}$ activity in cryosections at subcellular resolution. We emphasize that it is the TAMRA-FP labeling combined with immunohistochemistry and DAPI staining, each step utilizing different fluorophores with minimal wavelength overlap that makes tissue-ABPP a remarkably powerful new approach 


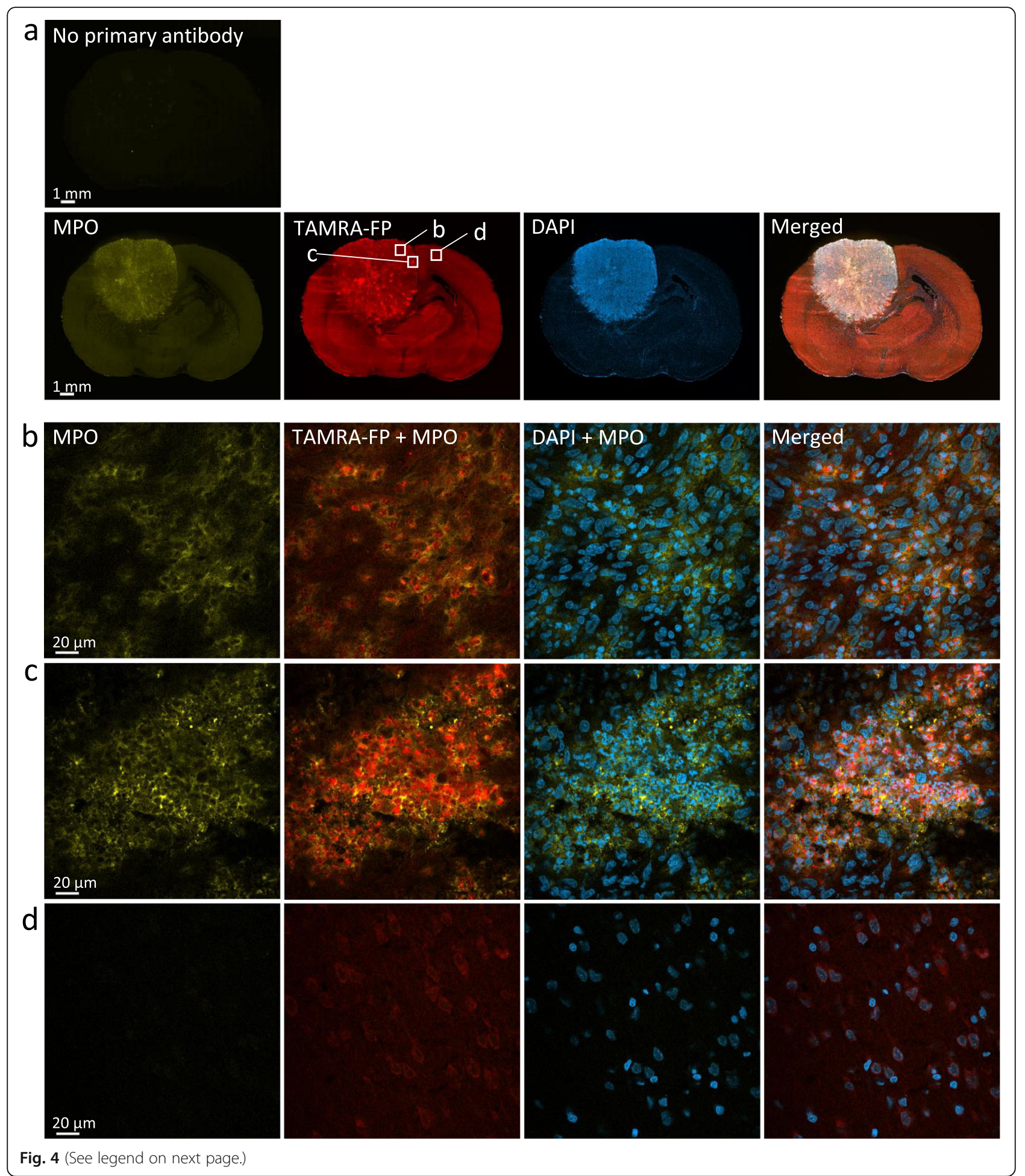

to portrait global $\mathrm{SH}$ activity in a complex proteome with preserved cyto-architecture.

The main focus of our studies was to achieve highresolution imaging of global $\mathrm{SH}$ activity throughout the glioma microenvironment, necessitating systematic immunohistochemical mapping of origins of $\mathrm{SH}$ activity and its localization with cell-type and tissue stiffness markers. Together with inhibitor-sensitivity of TAMRAFP labeling, these experiments provide the key validation for tissue-ABPP, ruling out the possibility that TAMRAFP binding would be nonspecific. Besides illuminating the complexity of tumor microenvironment, our study 
(See figure on previous page.)

Fig. 4 Confocal imaging of SH activity in relation to myeloperoxidase (MPO), a marker for neutrophils. Sections went through the tissue-ABPP protocol to label SHs (red) and were thereafter immunostained for MPO (yellow), followed by DAPI staining to visualize nuclei (blue). Panel a shows overall staining pattern throughout the coronal section plane. A control section undergoing identical staining protocol with no primary antibody is illustrated at top. Panel $\mathbf{b}$ shows staining pattern in glioma region characterized by intense SH activity originating from individual cells (TAMRA-FP hotspots). Panel $\mathbf{c}$ shows staining pattern in glioma region characterized by intense SH activity originating from cell clusters (TAMRAFP hotspot clusters). Panel $\mathbf{d}$ shows staining pattern in healthy brain (cortex). Note detectable expression of MPO in the glioma (a). Note MPOpositive cells in the region of TAMRA-FP hotspots and marked co-localization of MPO with the TAMRA-FP signal (b). Note abundance of MPOpositive cells within TAMRA-FP hotspot clusters, as well as close match of MPO staining with TAMRA-FP hotspot clusters (c). MPO is not visible in control cortical region (d). Primary antibody rabbit anti-MPO (Abcam, cat\# ab9535), dilution 1:25, secondary antibody Goat anti-rabbit IgG-Alexa Fluor 647 conjugate, dilution 1:100. Sections were from female rat 11. Scale bars: $1 \mathrm{~mm}$ in a, $20 \mu \mathrm{m}$ in b-d. Images were adjusted for brightness and contrast

unveiled heightened SH activity in glioma vs. normal brain and excluded major tumor-associated cells-types such as TAMs as a source of this activity. Unexpectedly, immunohistochemistry pinpointed TANs as the principal cell-type generating SH activity hotspots in glioma.

The activity probe that we used is commercially available and similar FP-probes have been previously used and extensively characterized for gel-based ABPP applications $[2,6,7]$. Furthermore, the coverage of $\mathrm{mSH}-$ family members recognized by the FP-probes is notable (>80\%) [37]. It could be argued that the use of broadspectrum probe complicates interpretation of signals emerging from cryosections and therefore quenched probes with limited reactivity would be preferable. However, such probes are not yet widely available and as our principal aim was to provide global portrait of $\mathrm{SH}$ activity within the tumor microenvironment, the use of broad-spectrum probe was fully justified. In analogy, the vast majority of gel-based ABPP studies prefer to utilize broad-spectrum probes in order to achieve the global $\mathrm{SH}$ activity profile of the studied proteome. However, tissue-ABPP studies in future could utilize also newlydeveloped activity probes, tailored to target a particular subset of $\mathrm{mSHs}$ [39].

SHs are key regulators of metabolic pathways in diseases including cancer, neurodegenerative diseases, metabolic syndrome and atherosclerosis. Tissue-ABPP offers a new tool to explore the myriad roles of SHs likely play in physiology and disease. We anticipate that tissue-ABPP should find broad immediate applications. We applied tissue-ABPP also successfully to spleen sections, mainly to provide additional validation in native immune tissue for TAMRA-FP labeling in relation to some of the antibodies (Figures S31 and S32). Previously, a single study has presented low-resolution TAMRA-FP imaging of AEBSF-sensitive SH activity in prostate cancer specimen in supplementary figure [40]. Although preliminary, that work extends the applicability of tissue-ABPP to human proteomes. As ABPP requires no a priori knowledge of the identity of the target, we envisage no imaginable reason why tissue-ABPP would not work for sections regardless of species and tissue source.
Both cell morphology and immunohistochemical evidence implicated neutrophils as the principal cell type generating TAMRA-FP hotspots and hotspot clusters in glioma. We emphasize that the hotspots described here represent $\mathrm{SH}$ activity, as TAMRA-FP labeling was sensitive to the $\mathrm{SH}$ inhibitors PMSF and DeBi-FP (Fig. 6). Furthermore, LC-MS/MS verified the presence of NSPs in the gel-pieces encompassing the intense $\sim 25 \mathrm{kDa}$ TAMRA-FP bands, allowing to conclude that NSPs account for the bulk of this activity. In further support are findings showing that the elastase-preferring V-DPP-probe detected TAMRAFP-sensitive $\sim 25 \mathrm{kDa}$ bands in glioma and that neutrophil and glioma proteomes shared similarly migrating intense $\mathrm{SH}$ bands with related pharmacology. Collectively these findings strongly suggest that NSPs rather than mSHs accounted for the TAMRA-FP hots spots and hotspot clusters.

The newly developed DPP-activity probes [13] and CTSG inhibitor Compound 22 [38] were designed to specifically target human NSPs. In our study, the compounds behaved as expected when tested using human proteomes (Figure S29 and data not shown). However, our cross-validation experiments clearly indicated that the Cy5-probes did not recognize mutual targets with TAMRA-FP in rat neutrophils or glioma. This was not totally unexpected, as both probe and inhibitor design exploited unique substrate recognition sequences around the catalytic sites which are not necessarily shared between human and rat NSPs. It is likely that the different warhead (DPP vs. FP) could also account for major differences in the reactivity and sensitivity of these probes to the inhibitors.

TANs are newcomers at the mainstage of cancer research [41]. In GBM, TANs seem to play mainly a tumorigenic role [42]. A recent study suggested involvement of TANs in GBM recurrence after radiotherapy by showing that neutrophils promoted cancer cells stemness [43]. In clinical samples, a positive correlation existed between neutrophils and patients diagnosed with recurrent tumor [43]. Future work could elucidate the role of TANs and NSPs in more detail. 


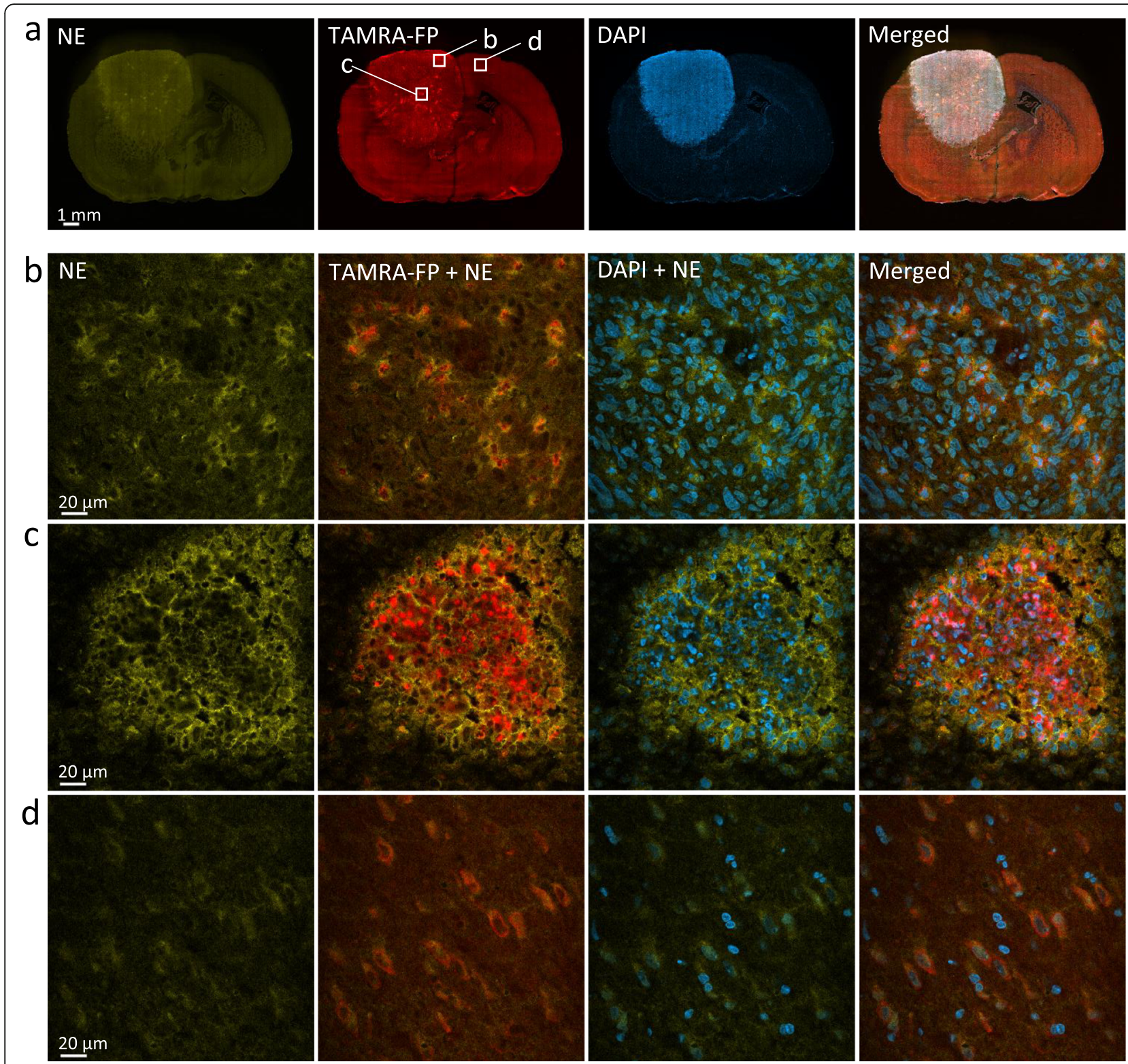

Fig. 5 Confocal imaging of SH activity in relation to neutrophil elastase (NE). Sections went through the tissue-ABPP protocol to label SHs (red) and were thereafter immunostained for NE (yellow), followed by DAPI staining to visualize nuclei (blue). Panel a shows overall staining pattern throughout the coronal section plane. A control section undergoing identical staining protocol with no primary antibody was not available for this experiment. Panel $\mathbf{b}$ shows staining pattern in glioma region characterized by intense SH activity originating from individual cells (TAMRA-FP hotspots). Panel c shows staining pattern in glioma region characterized by intense SH activity originating from cell clusters (TAMRA-FP hotspot clusters). Panel $\mathbf{d}$ shows staining pattern in healthy brain (cortex). Note detectable expression of NE throughout the glioma (a). Note in particular NE-positive cells in the region of TAMRA-FP hotspots and close match of NE-positive cells with the TAMRA-FP signal (b). Note strong NEimmunostaining of the TAMRA-FP hotspot clusters and close match of NE staining within the TAMRA-FP hotspot cluster (c). NE is not visible in control cortical region (d). Primary antibody rabbit anti-NE (Abcam, cat\# ab21595), dilution 1:500, secondary antibody Goat anti-rabbit IgG-Alexa Fluor 647 conjugate, dilution 1:100. Sections were from female rat 11. Scale bars: $1 \mathrm{~mm}$ in a, $20 \mu \mathrm{m}$ in b-d. Images were adjusted for brightness and contrast

Interestingly, the $\sim 30 \mathrm{kDa} \mathrm{SH}$ was enriched in glioma and was not detected in healthy brain or neutrophils. Collectively our tissue and gel-based ABPP experiments suggested that the majority of the MAFP-sensitive $\mathrm{SH}$ activity in glioma likely reflects activity of the $\sim 30 \mathrm{kDa}$ prominent SH band. LC-MS/MS disclosed PAFAH1b3 and PAFAH1b2, among others, in the gel-pieces encompassing the $\sim 25-30 \mathrm{kDa}$ TAMRA-FP bands. Noteworthy, these $\mathrm{mSHs}$ are MAFP-sensitive [8] and were proposed to play protumorigenic role in various cancers 


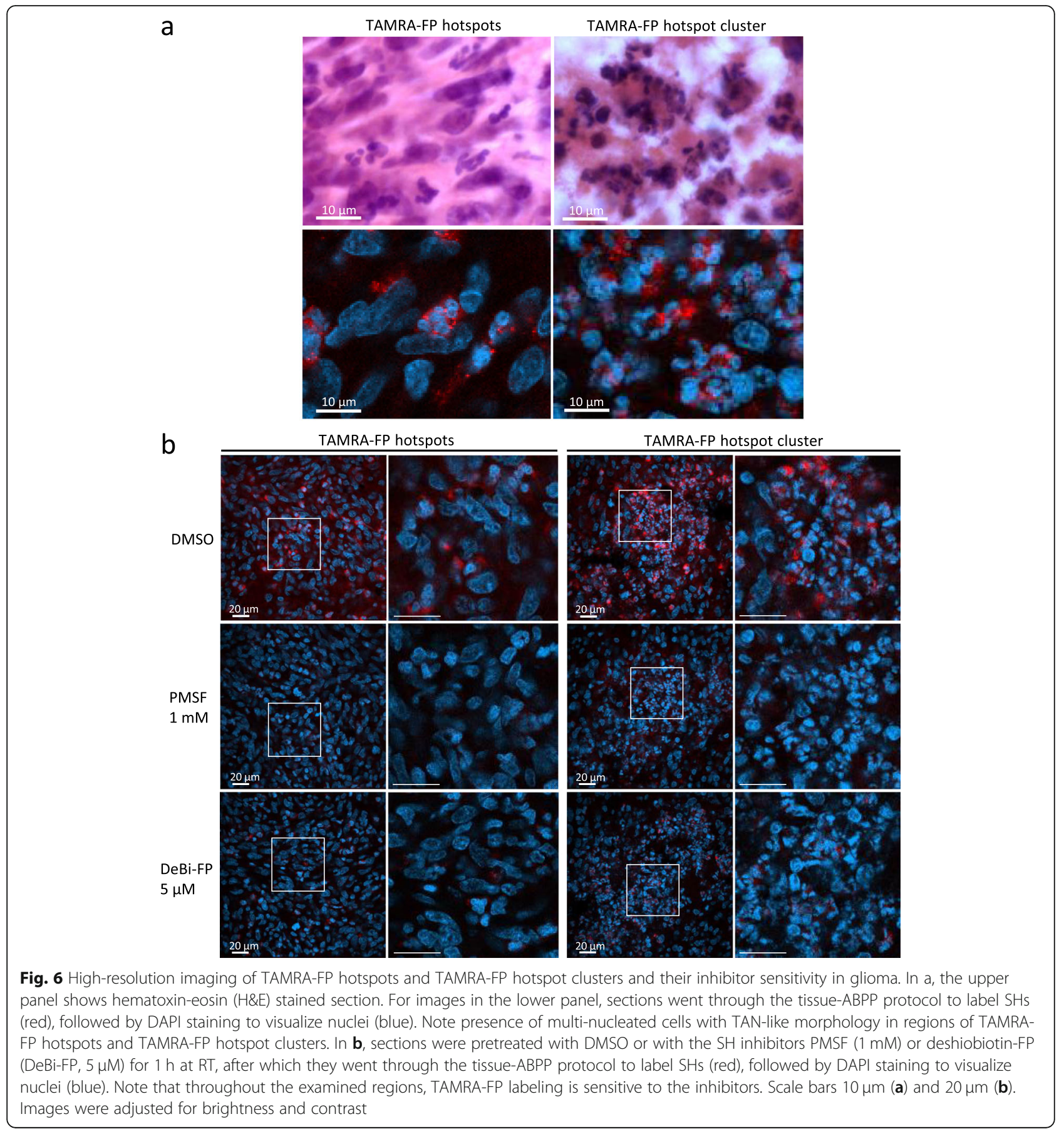

[44-46]. Future work should elucidate the role of PAFAH1b3 and PAFAH1b2 in glioma.

The presently used rat gliosarcoma model has been useful to test novel chemotherapeutic targeting strategies, antitumor effects of gene therapy, anti-angiogenic agents alone or in combination with radiation and chemotherapy ([18] and references therein). We chose this model for convenience as it was previously used by researchers at our university [29] and the expertise to execute such studies was readily available.
Gel- and MS-based ABPP has shown that isolated mouse brain primary cells such as neurons, astrocytes and microglia each show characteristic SH activity profiles [47]. Tissue-ABPP offers a powerful tool for detailed investigation of this issue in situ with all normal cell types present. Such studies should be of wide interest to increase knowledge on the myriad roles that SHs likely play in the CNS. Based on studies from this and other laboratories [14, 19-27], gel-ABPP using brain membranes typically detects $20-30 \mathrm{SH}$ bands, depending on 


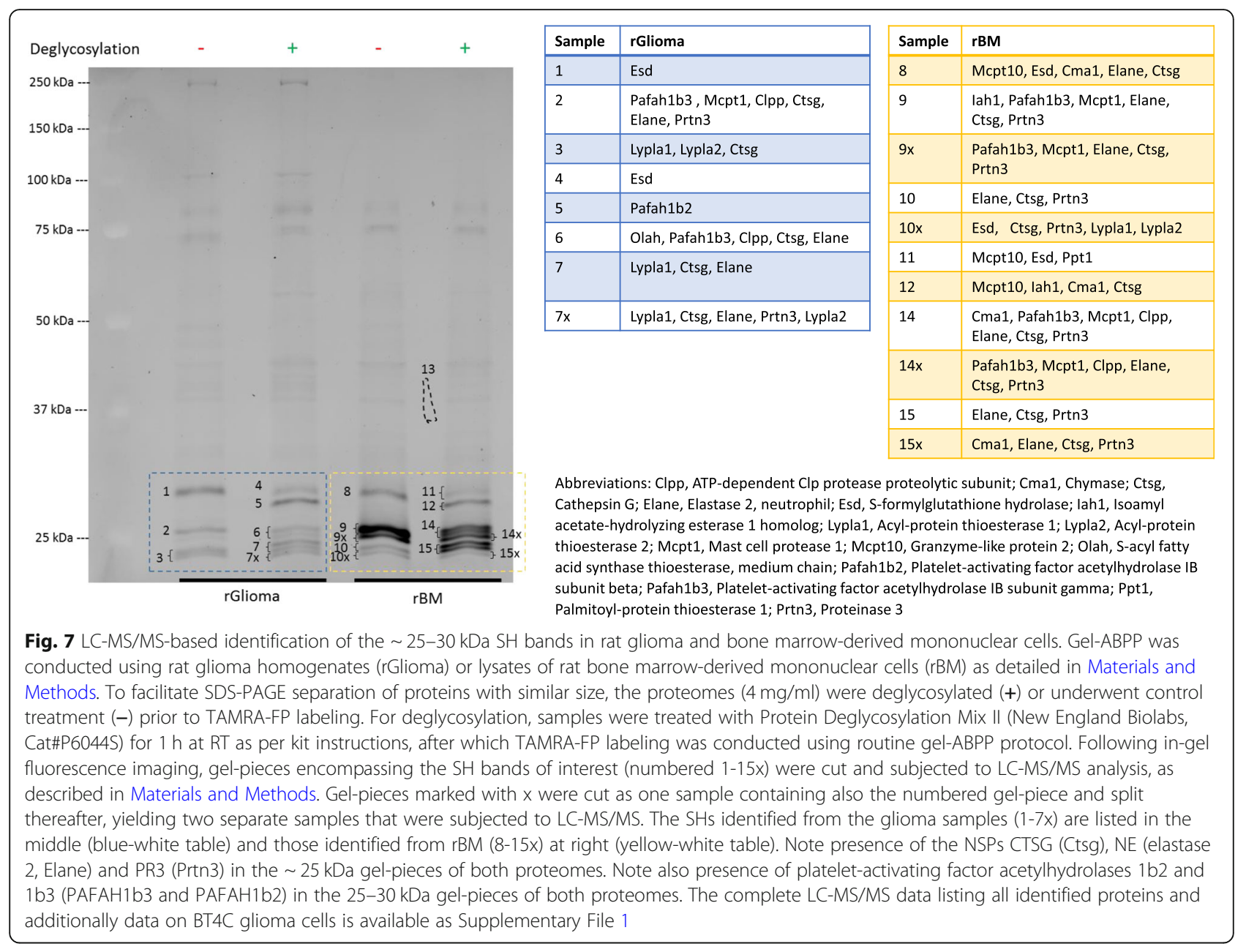

the brain region studied (see e.g. Figure 3). The band intensities vary, reflecting activities of the individual SHs. Weak or hardly detectable bands set the practical limit for sensitivity of gel-ABPP. As the majority of $\mathrm{mSH}$ are expressed in brain [1,2], only a fraction of these can be detected using gel-ABPP. By analogy, the situation is likely similar concerning sensitivity of tissue-ABPP. However, confocal imaging settings can be adjusted to optimally capture TAMRA-FP signal also from brain regions showing low fluorescence. Our study indicates that under the conditions employed, tissue-ABPP should offer adequate sensitivity to enable imaging of $\mathrm{SH}$ activity not only in the tumor microenvironment but also in different regions of the healthy brain (Figs. 1 and S33). A straightforward starting point could be comparative tissue-ABPP of an individual SH, e.g. the principal endocannabinoid hydrolase MAGL using brain sections of wild-type and MAGL-deficient mice, as MAGL knockout does not lead to compensatory changes in the activity of other SHs, as evidenced by gel-based ABPP [25]. Competitive tissue-ABPP would offer a complementary approach for sections where MAGL is chemically inactivated with highly selective and ultra-potent inhibitors, such as JJKK-048 and KML29 [3, 14].

\section{Conclusions}

Our study advances the ABPP approach to tissue sections, enabling high-resolution confocal fluorescence imaging of global $\mathrm{SH}$ activity in anatomically preserved complex native cellular environment. To achieve global portrait of SH activity throughout the tissue section, we used a probe with broad reactivity towards the SH family members. Thorough optimization and validation, complemented by gel-based ABPP, extensive immunohistochemical mapping enabling localization of $\mathrm{SH}$ hotspot activity, combined with LC-MS/MS-based target verification, collectively demonstrate that tissue-ABPP faithfully reports SH activity in cryosections. Tissue-ABPP is expected to provide sufficient degree of sensitivity, allowing profiling of not only heightened $\mathrm{SH}$ activity in the tumor, but also lower levels of SH activities in different regions of the healthy brain. As ABPP requires no a priori knowledge of the identity of the target, we envisage no imaginable reason why the presently described 
approach would not work for sections regardless of species and tissue source.

\section{Materials and Methods}

The principal aim of this study was to achieve highresolution imaging of global $\mathrm{SH}$ activity in glioma brain cryosections throughout the tumor microenvironment, necessitating systematic mapping of sources of $\mathrm{SH}$ activity by using cell-type specific immunohistochemical markers. To validate the tissue-ABPP approach, traditional gel-ABPP methodology combined with LC-MS/ MS-based target identification was employed.

\section{Chemicals and Reagents}

The following activity probes were used: TAMRA-FP (ActivX TAMRA-FP serine hydrolase probe, cat\# 88318) and DeBi-FP (ActivX Desthiobiotin-FP Serine Hydrolase Probe, cat\# 88317) were purchased from Thermo Scientific. The Cy5-labeled serine protease probes PK-DPP and V-DPP bearing a diphenylphosphonate (DPP) warhead [48] were used in selected experiments (Figures S27-28). Similarly, the Cy5-labeled NSP probes bearing a diphenylphosphonate (DPP) warhead [13] were used in selected experiments (Figure S29).

The following inhibitors were used: AEBSF (Sigma, cat\#A8456), PMSF (Sigma, cat\# P7626), IDFP (Cayman Chemical, cat\# 10215), MAFP (Sigma, cat\#M2939), CTSG-I (Calbiochem, cat\# 219372). Compound 22 was obtained from Joakim Swedberg (at that time in the Institute for Molecular Bioscience, University of Queensland, Brisbane, Australia).

For immunohistochemistry, various antibodies from different sources were used. The hyaluronan binding probe (bHABR) was prepared in-house [49] (Figure S13). The following secondary antibodies were used: Goat anti-rabbit IgG-Alexa Fluor 647 conjugate (Thermo Scientific, cat\# A-21245), Donkey anti-mouse IgG-Alexa Fluor 647 conjugate (Thermo Scientific, cat\# A-31571),

Donkey anti-goat IgG-Alexa Fluor 647 conjugate (Thermo Scientific, cat\# A-21447), and DyLight 488 Steptavidin (Vector Laboratories, cat\# SA-5488). Primary antibody source, dilution used in immunostaining and choice of secondary antibodies are given in the captions of the images showing immunostained sections (Figures S8-S26 and S31-S32). Nuclei were stained with DAPI (4',6-diamidino-2-phenylindole) (Sigma, cat\# D8417). The BSA used was essentially fatty acid free (Sigma cat\#A0281). All other chemicals were of highest purity available.

\section{Cell Culture}

BT4C cells were grown in Dulbecco's modified eagle's medium DMEM (EuroClone, high glucose, with stable
L-Glutamine, cat\# ECM0103L) supplemented with 10\% FBS (Euroclone, cat\# ECS0180L) in a humidified cell culture incubator with $5 \% \mathrm{CO}_{2}$ environment. The cells were maintained and sub-cultured two-three times a week at 1:6 splitting ratio. For preparation of the cell suspension for rat malignant glioma model, cells were trypsinized and collected in normal culture media (DMEM supplemented with 10\% FBS) followed by a brief centrifugation at $1000 \mathrm{rpm}$, for $4 \mathrm{~min}$ at $4{ }^{\circ} \mathrm{C}$, and re-suspended in fresh OPTI-MEM ${ }^{\circ}$ reduced serum medium (Gibco, cat\# 31985-062). Cells were then counted and diluted in the same medium to $2 \times 10^{6}$ cells $/ \mathrm{ml}$ so that each $5 \mu \mathrm{l}$ of cell suspension contained approximately 10,000 cells. For collecting cell pellets, cells were grown in several T-175 flasks until they become $95 \%$ confluent. On the day of collection, the cell culture media was aspirated from each flask, washed with cold 1xPBS, and cells were collected mechanically by using cell lifter/scraper in presence of $5 \mathrm{ml}$ of cold $1 \mathrm{x}$ PBS. Cell fractions collected from different flasks were then combined into a $50 \mathrm{ml}$ conical tube, washed with $1 \mathrm{xPBS}$, centrifuged at low speed $(300 \times \mathrm{g}$, for $10 \mathrm{~min}$ at $\left.4{ }^{\circ} \mathrm{C}\right)$ to obtain opaque cell pellet. The pellet was stored at $-80^{\circ} \mathrm{C}$.

\section{Animals \\ Malignant Rat Glioma Model}

In total 34 BDIX rats (18 males and 16 females, Envigo, Huntingdon, UK) weighing $127-267 \mathrm{~g}$ were used. The rats were anesthetized i.p. with ketamine (Ketalar ${ }^{\circ}, 60$ $\mathrm{mg} / \mathrm{kg}$ ) and medetomidine (Domitor ${ }^{\circ}, 0.4 \mathrm{mg} / \mathrm{kg}$ ) and placed in a stereotactic apparatus. After skin incision, a hole was drilled $1 \mathrm{~mm}$ posterior to the bregma and to the $2 \mathrm{~mm}$ right of sagittal suture. 10,000 BT4C tumor cells in $5 \mu \mathrm{l}$ of OPTI-MEM ${ }^{\circ}$ reduced serum medium were injected with a Hamilton syringe within right corpus callosum (depth $2.5-3.0 \mathrm{~mm}$ ). To avoid backflow, the injection was done slowly over $2-3 \mathrm{~min}$. The needle was left in place for $2 \mathrm{~min}$ and then slowly removed. Skin incision was closed with stiches followed by s.c. injections of antisedative (atipamezole, Antisedan ${ }^{\circ}, 1 \mathrm{mg} /$ $\mathrm{kg}$ ) and analgesic (carprofen, Rimadyl ${ }^{\circ}, 5 \mathrm{mg} / \mathrm{kg}$ ). Control rats were processed similarly, except that cells were omitted and only OPTI-MEM ${ }^{\circ}$ reduced serum medium was inoculated.

\section{Tissue Harvesting from Glioma Animals}

The rats were sacrificed 28-38 days after inoculation of the cells (the rats bearing tumors 28-31 days after inoculation). Animals were stunned with $\mathrm{CO}_{2}$ and then transcardially perfused with PBS. From majority of the rats, the whole brain was removed, dipped briefly in cold $\left(-80^{\circ} \mathrm{C}\right)$ isopentane and stored on dry ice. From six rats in total, the tumor and corresponding brain piece from 
contralateral cerebral hemisphere were separated and were frozen on dry ice. For bone marrow collection, total of four bones (Tibia, Femur, Humerus, and Radius) were collected from each animal and stored in cold $1 \mathrm{x}$ PBS for further processing.

\section{Control Rats Used for Harvesting Immune Cells and Tissue}

For collecting rat spleens, cerebella, and fresh blood as the source of neutrophils, 10-12 week-old male Rcc:Han WIST rats (Laboratory Animal Centre, University of Eastern Finland) were used. Rats were decapitated and blood was collected with a funnel into a $50 \mathrm{ml}$ conical tube soaked with EDTA $(1.5-1.8 \mathrm{mg} / \mathrm{ml}$ of fresh blood). The blood was then processed within $2 \mathrm{~h}$ of collection. The cerebella were dissected, dipped briefly in cold ($80^{\circ} \mathrm{C}$ ) isopentane and stored on dry ice. The spleens were collected, frozen on dry ice and then stored at $80^{\circ} \mathrm{C}$.

\section{Mice Used in Preliminary Tissue-ABPP Experiments}

To test fixatives and TAMRA-FP concentrations during tissue-ABPP method optimization (Figures S4-S5), 4week-old male JAXC57BL/6 J mice (Laboratory Animal Centre, University of Eastern Finland) were used. Mice were decapitated, the whole brain was removed, dipped briefly in cold $\left(-80^{\circ} \mathrm{C}\right)$ isopentane and frozen on dry ice and then stored at $-80^{\circ} \mathrm{C}$.

\section{MRI}

Magnetic resonance imaging (MRI) was used to verify tumor existence 12-14 and 22-24 days after inoculation of the cells. Anesthesia was induced with $5 \%$ isoflurane in a mixture of $70: 30 \% \mathrm{~N}_{2} \mathrm{O}: \mathrm{O}_{2}$ and was maintained at $1.5 \%$ isoflurane. MRI scanning was done with a $7 \mathrm{~T}$ Bruker PharmaScan system and ParaVision ${ }^{\circ} 5.1$ software (Bruker, Billerica, MA, USA). Turbo-RARE imaging sequence with the following parameters was used: TE 12.5 $\mathrm{ms} / 50 \mathrm{~ms}$, TR $4000 \mathrm{~ms}$, RARE factor 8 , FOV $2 \mathrm{~cm}$, $256 \times 256$ matrix, slice thickness $1 \mathrm{~mm}$ with 15 slices. The images were processed with a home-build Matlab program Aedes (University of Eastern Finland).

\section{Cryosectioning}

Coronal rat brain sections, horizontal mouse brain sections and rat spleen sections $(20 \mu \mathrm{m}$ thick) were cut at $19^{\circ} \mathrm{C}$ to $-21^{\circ} \mathrm{C}$ using a Leica cryostat (Leica Biosystems, IL, USA). The sections ( 3 sections per slide) were thawmounted onto Superfrost ${ }^{\circ}$ Plus slides (Menzel-Gläser, Germany), dried for $1-3 \mathrm{~h}$ at RT under a constant stream of air and stored thereafter at $-80^{\circ} \mathrm{C}$. Rat and mouse brain sections were cut according to Rat Brain Atlas (Paxinos \& Watson 1998).

\section{Tissue-ABPP}

Tissue-ABPP was used either for imaging SH activity alone or in combination with immunohistochemistry and nuclear staining, as detailed below.

\section{Protocol for Imaging SH Activity}

The assay was performed at RT $\left(20-22^{\circ} \mathrm{C}\right)$ unless otherwise stated. Generally, the fixing and all washing steps were performed in large volume $(\sim 200 \mathrm{ml})$ by dipping the slides into indicated buffer. Other steps of the protocol were performed by pipetting the indicated volume of buffer on the sections and by incubating the slides in a humidified chamber placed on a low-speed horizontal shaker.

The slides ( 3 tissue sections per slide) were thawed under a constant stream of air and thereafter each section was lined with a mini PAP pen (Fisher Scientific, cat\# 10464573). The slides were fixed with $4 \%$ paraformaldehyde (PFA) supplemented with $0.01 \%$ glutaraldehyde in $0.1 \mathrm{M}$ phosphate buffer ( $\mathrm{pH} 7.4$ ) for $10 \mathrm{~min}$ and rinsed thereafter $2 \times 5 \mathrm{~min}$ in $1 \times \mathrm{PBS}$. The assay protocol consisted of preincubations $2 \times 10 \mathrm{~min}$ in the assay buffer $(250 \mu \mathrm{l} /$ section $)$ containing $50 \mathrm{mM}$ Tris- $\mathrm{HCl}, \mathrm{pH} 7.4$; $1 \mathrm{mM}$ EDTA; $100 \mathrm{mM} \mathrm{NaCl} ; 5 \mathrm{mM} \mathrm{MgCl}_{2}$ and $0.1 \%$ (w/ v) BSA, followed by $60 \mathrm{~min}$ incubation in the assay buffer $(250 \mu \mathrm{l} /$ section $)$ in the presence of inhibitors or $1 \%$ (v/v) DMSO as a solvent. After washes $3 \times 1 \mathrm{~min}$ in the assay buffer $(250 \mu \mathrm{l} /$ section), the slides were incubated for $60 \mathrm{~min}$ with TAMRA-FP in the assay buffer $(95 \mu \mathrm{l} /$ section, routinely $0.5 \mu \mathrm{M}$ final concentration) and thereafter washed $3 \times 10 \mathrm{~min}$ in $0.1 \mathrm{M}$ phosphate buffer $(\mathrm{pH}$ 7.4). In some experiments, $\mathrm{Cy}-5$ labelled probes were used instead of TAMRA-FP as detailed in Figures S28 and S29. In preliminary experiments, presented in Figures S1 and S4-S5, the slides were dried under constant stream of air and imaged thereafter using a Fuji gel scanner. In subsequent experiments, the slides were further stained with nuclear stain DAPI $(2 \mu \mathrm{g} / \mathrm{ml}$ in $0.1 \mathrm{M}$ phosphate buffer, $300 \mu \mathrm{l} /$ section) for $15 \mathrm{~min}$ at $37^{\circ} \mathrm{C}$ and washed $2 \times 5$ min with $0.1 \mathrm{M}$ phosphate buffer $(\mathrm{pH} 7.4)$. Finally, the slides were embedded with Vectashield ${ }^{\circ}$ Antifade mounting medium (cat\# H-1000, Vector laboratories, CA, USA).

\section{Tissue-ABPP Combined with Immunohistochemistry and Nuclear Staining}

When immune markers were used, the preceding protocol for TAMRA-FP labeling is the same as described above. The TAMRA-FP labeled sections were washed $5 \times 5 \mathrm{~min}$ with $1 \mathrm{xPBS}$ supplemented with $1 \%(\mathrm{w} / \mathrm{v})$ BSA $(210 \mu \mathrm{l} /$ slide). The sections were incubated overnight with primary antibody $(195 \mu \mathrm{l} /$ slide $)$ at $4{ }^{\circ} \mathrm{C}$, washed $3 \times$ 10 min with $0.1 \mathrm{M}$ phosphate buffer ( $\mathrm{pH}$ 7.4) followed by $60 \mathrm{~min}$ incubation with secondary antibody $(195 \mu \mathrm{l} /$ 
slide). Both primary and secondary antibodies were diluted in $1 \times$ PBS supplemented with $1 \%(\mathrm{w} / \mathrm{v}) \mathrm{BSA}$. Finally, the slides were washed $3 \times 5 \mathrm{~min}$ with $0.1 \mathrm{M}$ phosphate buffer ( $\mathrm{pH} 7.4)$, stained with DAPI $(2 \mu \mathrm{g} / \mathrm{ml}$, $300 \mu \mathrm{l} /$ slide) for $15 \mathrm{~min}$ at $37^{\circ} \mathrm{C}$, washed $2 \times 5 \mathrm{~min}$ with $0.1 \mathrm{M}$ phosphate buffer ( $\mathrm{pH} 7.4$ ) and embedded with Vectashield $^{\circ}$ Antifade mounting medium.

\section{Histology}

To visualize tumor morphology, the sections were fist fixed with $4 \%$ PFA supplemented with $0.01 \%$ glutaraldehyde and then stained with Mayer's hematoxylin and eosin. Imaging was performed with a Zeiss Axio Imager M2 microscope (Carl Zeiss Microimaging, Jena, Germany).

\section{Fluorescence Imaging \\ Fluorescence Scanning}

For gel imaging and overview of tissue-ABPP slides, ChemiDoc $^{\text {Th }}$ MP imaging system (Bio-Rad, Hercules, CA, USA) was used. To visualize TAMRA-FP, Cy3 blot application (602/50, Green Epi) was used. For Cy5 probes or immunostained sections, Cy5 blot application (700/ 50, Red Epi) was used. In the early phase of this study (Figures S1, S4-S5), TAMRA-FP-labeled sections were imaged using Fuji gel scanner $\left(\lambda_{\text {ex }} 552 \mathrm{~nm} / \lambda_{\mathrm{em}} 575 \mathrm{~nm}\right)$.

\section{Confocal Microscopy}

The confocal microscope images were obtained with a Zeiss Axio Observer inverted microscope (10x, 40x (oil) or 63x (oil) -objectives) equipped with LSM800 confocal module (Carl Zeiss Microimaging GmbH, Jena, Germany). TAMRA-FP, DAPI, and secondary antibodies were imaged with $561 \mathrm{~nm}\left(\lambda_{\mathrm{ex}} 543 \mathrm{~nm} / \lambda_{\mathrm{em}} 567 \mathrm{~nm}\right), 405$ $\mathrm{nm}\left(\lambda_{\text {ex }} 353 \mathrm{~nm} / \lambda_{\text {em }} 465 \mathrm{~nm}\right)$, and $640 \mathrm{~nm}\left(\lambda_{\text {ex }} 653 \mathrm{~nm} /\right.$ $\lambda_{\mathrm{em}} 668 \mathrm{~nm}$ ) lasers, respectively. Secondary antibody used with bHABR (Figure S13) was imaged with $488 \mathrm{~nm}$ $\left(\lambda_{\text {ex }} 495 \mathrm{~nm} / \lambda_{\text {em }} 519 \mathrm{~nm}\right)$ laser. ZEN 2.3 (Blue) and ZEN 2.3 lite softwares (Carl Zeiss Microimaging $\mathrm{GmbH}$ ) were utilized for image processing and image analysis.

\section{Rat Bone Marrow Cell Isolation}

For bone marrow collection, total four bones (Tibia, Femur, Humerus, and Radius) were collected from each animal removing extra materials covering these bones, and stored in cold 1xPBS. The tip of each bone was cut with scissors, and flushed into a $50 \mathrm{ml}$ conical tube with ice-cold 1xPBS using a 25G-needle, until no red color was visible. The sample was then homogenized using a pipette, filtered through Corning ${ }^{\circ}$ cell strainer (CORNING, cat\# 431750, $40 \mu \mathrm{m}$ nylon), and centrifuged at $443 \times \mathrm{g}$ for $4 \mathrm{~min}$ at $4{ }^{\circ} \mathrm{C}$ to pellet the cells, and suspended in $4 \mathrm{ml}$ of $1 \times$ PBS. Four $\mathrm{ml}$ of ice cold ficoll-paque plus (GE Healthcare, 17-1440-02, density $1.077 \pm 0.001 \mathrm{~g} / \mathrm{ml}$ ) was pipetted into a $10 \mathrm{ml}$ transparent tube, and bone marrow cells $(4 \mathrm{ml})$ were then overlaid on top of ficollpaque plus carefully avoiding mixing of the two layers, then centrifuged at $443 \times \mathrm{g}, 20^{\circ} \mathrm{C}$ for $40 \mathrm{~min}$ withoutbrake in a swinging bucket centrifuge. The mononuclear cells were collected from the interface of PBS (top layer), and Ficoll-Red blood cells with granulocytes (two bottom layers). The cells were then washed by adding 3-6 volumes of $1 \mathrm{xPBS}$ followed by centrifugation at $1560 \times \mathrm{g}$ for $4 \mathrm{~min}$ at $4{ }^{\circ} \mathrm{C}$ to remove extra Ficoll. The solid-white pellets were then stored at $-80^{\circ} \mathrm{C}$.

\section{Human and Rat Blood Neutrophil Isolation}

For the isolation of neutrophils from human and rat blood, we used ficoll-paque plus (GE Healthcare, cat\# 17-1440-02, Density $1.077 \pm 0.001 \mathrm{~g} / \mathrm{ml}$ ) density gradient protocol adapted from [50,51]. Human and rat samples were handled separately. Briefly, fresh blood samples were collected from 5 rats $(\sim 33 \mathrm{ml})$ and a human donor $(\sim 12 \mathrm{ml})$ in sodium EDTA $(1.5-1.8 \mathrm{mg} / \mathrm{ml}$ of fresh blood). Five $\mathrm{ml}$ of blood was overlaid carefully on $5.0 \mathrm{ml}$ of ficoll-paque plus in a $15 \mathrm{ml}$ conical tube, centrifuged at $500 \times \mathrm{g}$ for $35 \mathrm{~min}$ at RT with brake off. The granulocyte-rich pellet was collected by removing three upper layers (plasma, mononuclear cells, and remaining ficoll-paque plus), and processed further by preparing $1 \mathrm{x}$ HBSS-buffer (LONZA, BioWhittaker, cat\# 04-315Q) suspension, 3\% (w/v) dextran (Dextran T500, Pharmacia, cat\# 17-0320-01, 450,000-550,000 M.W.) sedimentation, collecting granulocyte-enriched supernatant to a new tube; sample was centrifuged $(300 \times \mathrm{g}$ for $10 \mathrm{~min}$ at RT) and after removing the supernatant, remaining erythrocytes were lysed using hypotonic buffer, re-equilibrated to restore isotonicity, followed by centrifugation $(300 \times \mathrm{g}$ for $10 \mathrm{~min}$ at RT). Final washing and centrifugation (300 $\times \mathrm{g}$ for $10 \mathrm{~min}$ at RT) was done using HBSS buffer, resulting to clear white pellet. The pellet was stored at $80^{\circ} \mathrm{C}$.

\section{Preparation of Proteomes for Gel-Based ABPP}

Cell pellet was thawed in ice, and $100-500 \mu \mathrm{l}$ of $1 x \mathrm{PBS}$ was added to each tube depending on the size of the pellet (approximately $1 \mathrm{~mm}$-sized pellet in height, was resuspended in $100 \mu \mathrm{l}$ of $1 \mathrm{xPBS}$ ), mixed properly, spun, and went through freeze-thaw lysis procedure for at least five cycles (freezing at $-80^{\circ} \mathrm{C}$ for $15 \mathrm{~min}$ or on dry ice for $5 \mathrm{~min}$, thawing at $37^{\circ} \mathrm{C}$ water bath for $1 \mathrm{~min}$ ). Tissue samples were thawed in ice, weighted and required amount of $50 \mathrm{mM}$ Tris- $\mathrm{HCl}$, pH 7.4 + $150 \mathrm{mM} \mathrm{NaCl}$ buffer was added based on $1 \mathrm{ml}$ volume per $1 \mathrm{~g}$ wet tissueratio, homogenized in glass-glass homogenizer in ice. The samples were collected into separate $2 \mathrm{ml}$ Eppendorf tubes after washing with double volume of the Trisbuffer used initially. Finally, homogenized tissue samples 
and freeze-thaw-treated cell suspensions were sonicated on ice to obtain completely homogenized samples. Samples were then aliquoted, protein concentration was determined using BCA-200 Protein Assay Kit (PIERCE, 23226) as per company's protocol, and stored at $-80^{\circ} \mathrm{C}$. Rat cerebellar membranes were prepared as previously described [52].

\section{Competitive Gel-Based ABPP}

Competitive gel-ABPP was conducted using the Cy3labeled probe TAMRA-FP or in selected experiments (Figures S28 and S29) various Cy5-labeled serine protease probes, following general outlines of our previous publication [53]. Briefly, $25 \mu \mathrm{l}$ of proteomes $(5-10 \mu \mathrm{g}$ protein) diluted in PBS were preincubated with the indicated inhibitors (50-fold desired final concentration) or a vehicle (DMSO) for $1 \mathrm{~h}$ at $\mathrm{RT}$, followed by addition of activity probe (in the case of TAMRA-FP, final concentration was $1 \mu \mathrm{M})$. The reaction was quenched by adding 2xSDS-loading buffer, followed by protein separation by SDS-PAGE (10\%). For some lanes, Precision Plus Protein $^{\text {tx }}$ standards (BIO-RAD, cat\# 161-0373) were included. In early phase of this study, TAMRA-FP fluorescence was imaged using Fujifilm FLA-3000 laser fluorescence scanner (Fujifilm, Tokyo, Japan) (Fluor. $532 \mathrm{~nm}$; Filter: O580 nm). Thereafter, ChemiDoc $^{\mathrm{Tm}} \mathrm{MP}$ imaging system (BIO-RAD, Hercules, California, USA) was used as follows: Cy3 blot application (602/50, Green Epi, Manual Exposure 10s-120s) was used, and Cy5 blot application (700/50, Red Epi, Manual Exposure 1 s) was used to image MW markers and all Cy5-labelled probes.

\section{Sample Preparation for LC-MS/MS}

Gel casting and ABPP were done with special care in order to minimize potential contamination by foreign proteins. Gel electrophoresis and imaging was as described above. As TAMRA-FP labelling is visible only in imager under specific wavelength, the cutting of intended bands was done by keeping gel in a new, clear square BioAssay Dish (Corning, cat\# 431111, $245 \mathrm{mmx}$ $245 \mathrm{~mm}$ ) put on a horizontally positioned computer screen where the imaged picture was opened and positioned accurately (as accurate as possible). The gel pieces was cut using scalpel no. 11 and collected into labelled $1.5 \mathrm{ml}$ Eppendorf tube. The cut gel was then imaged again to check the accuracy of sample collection (Supplementary File 1_LC-MS-MS data). Collected samples were then wrapped with Parafilm ${ }^{\circ}$, stored in $-80^{\circ} \mathrm{C}$ freezer, and shipped to Helsinki proteomics lab in dry ice contained box.

\section{LC-MS/MS Analysis}

Protein bands were cut out of the polyacrylamide gel (Bio-Rad, USA) and "in-gel" digested cystein bonds were reduced with $0,045 \mathrm{M}$ dithiothreitol (Sigma, cat\# D0632) for $20 \mathrm{~min}$ at $37^{\circ} \mathrm{C}$ and alkylated with $0,1 \mathrm{M}$ iodoacetamide (Sigma-Aldrich, cat\# 57670) at room temperature. Samples were digested by adding $0,75 \mu \mathrm{g}$ trypsin (Sequencing Grade Modified Trypsin, V5111, Promega). After digestion peptides were purified with $\mathrm{C} 18$ microspin columns (Harvard Apparatus) according to manufactures protocol and re-dissolved in $30 \mu \mathrm{l}$.

Liquid chromatography coupled to tandem mass spectrometry (LC-MS/MS) analysis was carried out on an EASY-nLC1000 (Thermo Fisher Scientific, Germany) connected to a Velos Pro-Orbitrap Elite hybrid mass spectrometer (Thermo Fisher Scientific, Germany) with nano electrospray ion source (Thermo Fisher Scientific, Germany). The LC-MS/MS samples were separated using a two-column setup consisting of a $2 \mathrm{~cm} \mathrm{C18-A1}$ trap column (Thermo Fisher Scientific, Germany), followed by $10 \mathrm{~cm}$ C18-A2 analytical column (Thermo Fisher Scientific, Germany). The linear separation gradient consisted of $5 \%$ buffer B in $5 \mathrm{~min}, 35 \%$ buffer B in $60 \mathrm{~min}, 80 \%$ buffer B in $5 \mathrm{~min}$ and $100 \%$ buffer B in 10 min at a flow rate of $0,3 \mu \mathrm{l} / \mathrm{min}$ (buffer A: $0,1 \%$ TFA in $1 \%$ acetonitrile; buffer B: $0,1 \%$ TFA acid in $98 \%$ acetonitrile). $6 \mu \mathrm{l}$ of sample was injected per LC-MS/MS run and analyzed. Full MS scan was acquired with a resolution of 60,000 at normal mass range in the orbitrap analyzer the method was set to fragment the 20 most intense precursor ions with CID (energy 35). Data was acquired using LTQ Tune software.

Acquired MS2 scans were searched against Rattus norvegicus protein data-base using the Sequest search algorithms in Thermo Proteome Discoverer. Allowed mass error for the precursor ions was $15 \mathrm{ppm}$. And for the fragment in $0,8 \mathrm{Da}$. A static modification parameter was set for carbamidomethyl $+57,021 \mathrm{Da}(\mathrm{C})$ of cysteine residue. Methionine oxidation $(+15,995 \mathrm{Da}(\mathrm{M}))$ and TAMRA-FP (+659,312 Da (S, Y)) were set as dynamic modifications. Only full-tryptic peptides were allowed for scoring maximum of 1 missed cleavages were considered.

\section{Supplementary information}

Supplementary information accompanies this paper at https://doi.org/10. 1186/s12575-020-00118-4.

\footnotetext{
Additional file 1 : Figure S1. Activity-based protein profiling (ABPP) and the power of this approach to unveil SH activity in glioma. Figure S2. Characteristics of the rat BT4C gliosarcoma model. Figure S3. Coronal plane MRI images of glioma and control brains. Figure S4. Testing various fixation method for rodent brain sections. Figure S5. Effect of TAMRA-FP concentration on fluorescence signal in mouse brain sections. Figure S6. Effects of $\mathrm{pH}$ and buffer composition on TAMRA-FP signal and its inhibitor sensitivity. Figure S7. Comparative gel-based ABPP of seven animals confirming distinct $\mathrm{SH}$ activity profiles between glioma and control brain. Figure S8. Confocal imaging of SH activity in relation to proliferating tumor cells. Figure $\mathbf{S 9}$. Confocal imaging of SH activity in
} 
relation to astrocytes. Figure $\mathbf{S 1 0}$. Confocal imaging of SH activity in relation to blood vessels. Figure S11. Confocal imaging of SH activity in relation to heme oxygenase 1 (HO-1). Figure S12. Confocal imaging of $\mathrm{SH}$ activity in relation to microglial marker Iba1. Figure S13. Confocal imaging of $\mathrm{SH}$ activity in relation to hyaluronan (HA). Figure S14. Confocal imaging of SH activity in relation to HA receptor CD44. Figure S15. Confocal imaging of $\mathrm{SH}$ activity in relation to the stiffness marker pMLC2. Figure S16. Confocal imaging of SH activity in relation to the stiffness marker tenascin C. Figure S17. Confocal imaging of SH activity in relation to CD45, a marker for nucleated hematopoietic cells. Figure S18. Confocal imaging of $\mathrm{SH}$ activity in relation to $\mathrm{CD} 11 \mathrm{~b} / \mathrm{c}$, a marker for phagocytes. Figure S19. Confocal imaging of SH activity in relation to CD68, a marker for monocytes and macrophages. Figure S20. Confocal imaging of SH activity in relation to CD163, a marker for monocytes and macrophages. Figure S21. Confocal imaging of SH activity in relation to CD169, a marker for macrophages. Figure S22. Confocal imaging of SH activity in relation to T cell marker CD4. Figure S23. Confocal imaging of SH activity in relation to T cell marker CD8. Figure S24. Confocal imaging of SH activity in relation to FcERly, a marker for mast cells, eosinophils, basophils and monocytes. Figure S25. Confocal imaging of SH activity in relation to chymase (CMA1), a marker for mast cells. Figure S26. TAMRA-FP signal at the site of injection in sham-operated animals. Figure S27. Gel-ABPP of rat glioma proteomes using Cy5-labeled serine protease activity probes PK-DPP and V-DPP. Figure S28. Tissue-ABPP of glioma sections using Cy5-labeled activity probes PK-DPP and V-DPP. Figure S29. ABPP of rat neutrophil and glioma samples using Cy5-labeled neutrophil serine protease (NSP) probes in combination with TAMRA-FP. Figure S30. Inhibitor profiles of human cathepsin G (hCTSG) and the prominent 25-30 kDa SH bands in rat bone-marrow-derived mononuclear cells and neutrophils. Figure S31. High-resolution imaging of TAMRA-FP hotspots and their inhibitor sensitivity in rat spleen. Figure S32. Confocal imaging of $\mathrm{SH}$ activity in rat spleen sections in relation to selected immunomarkers. Figure $\mathbf{S 3 3}$. Tissue-ABPP offers sufficient sensitivity to enable imaging of TAMRA-FP fluorescence in regions of the healthy brain.

Additional file $\mathbf{2}$ Video $\mathbf{S 1}$. 3D-animation of merged TAMRA-FP-DAPI fluorescence throughout the section thickness in TAMRA-FP hotspots and TAMRA-FP hotspot clusters (related to Fig. 1).

Additional file 3. Complete LC-MS/MS data of all proteins identified from the ABPP gel-pieces.

\section{Abbreviations}

ABHD: $\alpha / \beta$-hydrolase domain-containing; ABPP: Activity-based protein profiling; ABPP-MudPIT: A platform combining ABPP and multidimensional protein identification techniques; AEBSF: Aminoethylbenzenesulfonyl fluoride; APT1/APT2: Acyl-protein thioesterase 1/2; BBB: Blood-brain-barrier;

BSA: Bovine serum albumin; CD: Cluster of differentiation; CTSG: Cathepsin G; CTSG-I: CTSG inhibitor; DAPI: 4',6-diamidine-2'-phenylindole; DeBi-

FP: Desthiobiotin-FP; DPP: Diphenylphosphonate; EnPlex: A high-throughput platform based on ABPP; FAAH: Fatty acid amide hydrolase; FASN: Fatty acid synthase; FP: Fluorophosphonate; GBM: Glioblastoma multiforme; GFAP: Glial fibrillary acidic protein; HA: Hyaluronan; HO-1: Heme oxygenase-1; IDFP: Isopropyl dodecylfluorophosphonate; LC/MS-MS: Liquid chromatography coupled to tandem mass spectrometry; LYPLA1/ 2: Lysophospholipase A1/A2; MAFP: Methyl arachidonyl fluorophosphonate; MAGL: Monoacylglycerol lipase; MPO: Myeloperoxidase; mSH: Metabolic serine hydrolase; NCEH1: Neutral cholesteryl ester hydrolase 1; NE: Neutrophil elastase (NE); NSP: Neutrophil serine protease; NSP4: Neutrophil proteinase 4; PAFAH1b2/1b3: PAF-acetylhydrolase 1b2/1b3; pMLC2: Phosphorylated myosin light chain 2; PMSF: Phenylmethylsulfonyl fluoride; PR3: Proteinase 3; PREP/POP: Prolyl oligopeptidase; rBM: Rat bone-marrow-derived mononuclear cells; SDS-PAGE: Sodium dodecyl sulfate polyacrylamide gel electrophoresis; SH: Serine hydrolase; TAMRA-FP: Serine hydrolase probe; TAMs: Tumor-associated macrophages; TANs: Tumor-associated neutrophils

\section{Acknowledgements}

We thank Tanja Kosunen and Dina Navia-Paldanius for contributing to the pilot tissue-ABPP experiments and Miika Martikainen for practical guidance with glioma animals. We thank Mikko Kettunen and Kimmo Jokivarsi for help in MRI and Taina Vihavainen, Taija Hukkanen, Satu Marttila, Eija Rahunen, Shabana Azmy, Juha Niskanen for skillful technical help. We thank Anne Koivisto and Ilkka Harvima for enzymo-histochemical staining of tryptase and chymase. We thank Sini Miettinen and Tiina Öhman for help in analyzing and processing the LC-MS/MS data. We thank Joakim Swedberg (at that time in the Institute for Molecular Bioscience, University of Queensland, Brisbane, Australia) for providing compound 22. We are grateful for the UEF Cell and Tissue Imaging Unit and for Kuopio Biomedical Imaging Unit (Kuopio-BIU) for providing facilities for histology, confocal microscopy and MRI.

\section{Authors' Contributions}

J.T.L., T.W. and J.R.S. conceptualized the study. N.A., H.J., P.K.S., E.M. and S.K. carried out the in vitro experiments. N.A., P.K.S., T.W. and H.S. generated the glioma rats. P.K.S. and N.A. carried out MRI imaging of tumors, and harvesting of tissue from the animals. P.K.S. isolated bone-marrow cells and neutrophils, and performed cell culture and gel-based ABPP experiments. P.K.S. prepared samples and M.V. supervised the mass-spectrometry experiments. P.K.S. and J.R.S. analyzed the MS data. L.E.M. synthesized tryptase and elastase probes, P.K. and M.D. synthesized NSP probes. N.A. and H.J. carried out tissue-ABPP experiments. N.A., H.J., J.R.S. and J.T.L. designed tissue-ABPP experiments; N.A. and H.J. performed confocal fluorescence imaging with help and expertise provided by S.P-S. and K.R.. J.T.L., N.A. and P.K.S. wrote the manuscript. All others edited the manuscript. J.T.L. acquired the funding. The author(s) read and approved the final manuscript.

\section{Funding}

J.T.L. was supported by the Finnish Academy (grant no. 278212). L.E.M. was supported by a Grimwade Fellowship from the Russell and Mab Grimwade Miegunyah Fund at The University of Melbourne and a DECRA Fellowship from the Australian Research Council (ARC, DE180100418). Drag laboratory is supported by Foundation for Polish Science and National Science Centre in Poland. PK. is the beneficiary of a L'Oreal Poland and the Polish' Ministry of Science and Higher Education scholarships.

\section{Availability of Data and Materials}

The datasets supporting the conclusions of this article are included within the article and its additional files. Correspondence and request for materials should be addressed to J.T.L. or J.R.S., to P. K and M.D. (NSP probes) or to L.E.M. (elastase/tryptase probes).

\section{Ethics Approval and Consent to Participate}

All experimental procedures for animal studies have been approved by the Committee for the Welfare of Laboratory Animals of the University of Eastern Finland and by the National Animal Experiment Board (ELLA), and were performed in accordance with all national and local guidelines and regulations. Blood for neutrophil isolation was donated by one volunteer member of the research group.

\section{Consent for Publication}

All authors have read and approved the final manuscript.

\section{Competing Interests}

The authors declare that they have no competing interests.

\section{Author details}

${ }^{1}$ Institute of Biomedicine, University of Eastern Finland (UEF), POB 1627. Fl-70211 Kuopio, Finland. ${ }^{2}$ Aurealis Pharma, Kuopio, Finland. ${ }^{3}$ Institute of Biotechnology \& HiLIFE, University of Helsinki, Helsinki, Finland. ${ }^{4}$ Department of Biochemistry and Molecular Biology, Bio21 Molecular Science and Biotechnology Institute, The University of Melbourne, Parkville, VIC, Australia. ${ }^{5}$ Drug Discovery Biology, Monash Institute of Pharmaceutical Sciences, Monash University, Parkville, VIC, Australia. ${ }^{6}$ Department of Oral and Maxillofacial Surgery, New York University College of Dentistry, Bluestone Center for Clinical Research, New York, NY, USA. ${ }^{7}$ Department of Bioorganic Chemistry, Wroclaw University of Science and Technology, Wroclaw, Poland.

Received: 12 November 2019 Accepted: 19 February 2020

Published online: 15 March 2020

\section{References}

1. Long JZ, Cravatt BF. The metabolic serine hydrolases and their functions in mammalian physiology and disease. Chem Rev. 2011;111:6022-63. 
2. Simon GM, Cravatt BF. Activity-based proteomics of enzyme superfamilies: serine hydrolases as a case study. J Biol Chem. 2010;285:11051-5.

3. Bachovchin DA, Cravatt BF. The pharmacological landscape and therapeutic potential of serine hydrolases. Nat Rev Drug Discov. 2012;11:52-68 Available from: http://www.ncbi.nlm.nih.gov/pmc/articles/PMC3665514/.

4. Liu Y, Patricelli MP, Cravatt BF. Activity-based protein profiling: the serine hydrolases. Proc Natl Acad Sci U S A. 1999;96:14694-9.

5. Jessani N, Niessen S, Wei BQ, Nicolau M, Humphrey M, Ji Y, et al. A streamlined platform for high-content functional proteomics of primary human specimens. Nat Methods. 2005:2:691-7.

6. Nomura DK, Dix MM, Cravatt BF. Activity-based protein profiling for biochemical pathway discovery in cancer. Nat Rev Cancer. 2010;10:630-8.

7. Niphakis MJ, Cravatt BF. Enzyme inhibitor discovery by activity-based protein profiling. Annu Rev Biochem. 2014;83:341-77.

8. Bachovchin DA, Koblan LW, Wu W, Liu Y, Li Y, Zhao P, et al. A highthroughput, multiplexed assay for superfamily-wide profiling of enzyme activity. Nat Chem Biol. 2014;10:656-63 Available from: https://doi.org/10. 1038/nchembio.1578.

9. Edgington-Mitchell LE, Bogyo M, Verdoes M. Live cell imaging and profiling of cysteine cathepsin activity using a quenched activity-based probe. Methods Mol Biol. 2017;1491:145-59.

10. Withana NP, Garland M, Verdoes M, Ofori LO, Segal E, Bogyo M. Labeling of active proteases in fresh-frozen tissues by topical application of quenched activity-based probes. Nat Protoc. 2016;11:184-91.

11. Abd-Elrahman I, Kosuge H, Wises Sadan T, Ben-Nun Y, Meir K, Rubinstein C, et al. Cathepsin activity-based probes and inhibitor for preclinical atherosclerosis imaging and macrophage depletion. PLoS One. 2016;11: e0160522 Available from: https://doi.org/10.1371/journal.pone.0160522.

12. Liu Y, Walker E, lyer SR, Biro M, Kim I, Zhou B, et al. Molecular imaging and validation of margins in surgically excised nonmelanoma skin cancer specimens. J Med Imaging. 2019;6:16001.

13. Kasperkiewicz P, Altman Y, D'Angelo M, Salvesen GS, Drag M. Toolbox of fluorescent probes for parallel imaging reveals uneven location of serine proteases in neutrophils. J Am Chem Soc. 2017;139:10115-25.

14. Aaltonen N, Savinainen JR, Ribas CR, Rönkkö J, Kuusisto A, Korhonen J, et al. Piperazine and piperidine triazole ureas as ultrapotent and highly selective inhibitors of monoacylglycerol lipase. Chem Biol. 2013;20:379-90.

15. Sim LJ, Selley DE, Childers SR. In vitro autoradiography of receptor-activated $\mathrm{G}$ proteins in rat brain by agonist-stimulated guanylyl 5'-[gamma-[35S]thio]triphosphate binding. Proc Natl Acad Sci U S A. 1995;92:7242-6.

16. Ohgaki H, Kleihues P. Genetic alterations and signaling pathways in the evolution of gliomas. Cancer Sci. 2009;100:2235-41.

17. Claes A, Idema AJ, Wesseling P. Diffuse glioma growth: a guerilla war. Acta Neuropathol. 2007;114:443-58.

18. Barth RF, Kaur B. Rat brain tumor models in experimental neuro-oncology: the C6, 9L, T9, RG2, F98, BT4C, RT-2 and CNS-1 gliomas. J Neuro-Oncol. 2009;94:299-312 Available from: http:/www.ncbinlm.nih.gov/pmc/articles/ PMC2730996/

19. Casida JE, Nomura DK, Vose SC, Fujioka K. Organophosphate-sensitive lipases modulate brain lysophospholipids, ether lipids and endocannabinoids. Chem Biol Interact. 2008;175:355-64.

20. Hoover HS, Blankman JL, Niessen S, Cravatt BF. Selectivity of inhibitors of endocannabinoid biosynthesis evaluated by activity-based protein profiling. Bioorg Med Chem Lett. 2008;18:5838-41.

21. Adibekian A, Martin BR, Chang JW, Hsu K-L, Tsuboi K, Bachovchin DA, et al. Confirming target engagement for reversible inhibitors in vivo by kinetically tuned activity-based probes. J Am Chem Soc. 2012;134:10345-8.

22. Patel JZ, Parkkari T, Laitinen T, Kaczor AA, Saario SM, Savinainen JR, et al. Chiral 1,3,4-oxadiazol-2-ones as highly selective FAAH inhibitors. J Med Chem. 2013:56:8484-96.

23. Parkkari T, Haavikko R, Laitinen T, Navia-Paldanius $D$, Rytilahti $R$, Vaara $M$, et al. Discovery of triterpenoids as reversible inhibitors of $\alpha / \beta$-hydrolase domain containing 12 (ABHD12). PLoS One. 2014;9:e98286.

24. Savinainen JR, Patel JZ, Parkkari T, Navia-Paldanius D, Marjamaa JJT, Laitinen $T$, et al. Biochemical and pharmacological characterization of the human lymphocyte antigen B-associated transcript 5 (BAT5/ABHD16A). PLoS One. 2014;9:e109869 Available from: http://dx.plos.org/10.1371/journal.pone.01 09869.

25. Navia-Paldanius D, Aaltonen N, Lehtonen M, Savinainen JR, Taschler U, Radner FPW, et al. Increased tonic cannabinoid CB1R activity and brain region-specific desensitization of $\mathrm{CB} 1 \mathrm{R}$ Gi/o signaling axis in mice with global genetic knockout of monoacylglycerol lipase. Eur J Pharm Sci. 2015; 77:180-8.

26. Patel JZ, Nevalainen TJ, Savinainen JR, Adams Y, Laitinen T, Runyon RS, et al. Optimization of 1,2,5-thiadiazole carbamates as potent and selective ABHD6 inhibitors. Chem Med Chem. 2015;10:253-65.

27. Navia-Paldanius D, Patel JZ, López Navarro M, Jakupović H, Goffart S, Pasonen-Seppanen S, et al. Chemoproteomic, biochemical and pharmacological approaches in the discovery of inhibitors targeting human a/ß-hydrolase domain containing 11 (ABHD11). Eur J Pharm Sci. 2016;93: 253-63.

28. Lin DTS, Davis NG, Conibear E. Targeting the Ras palmitoylation/ depalmitoylation cycle in cancer. Biochem Soc Trans. 2017;45:913-21.

29. Wirth T, Ahmad F, Pacholska A, Samaranayake H, Ylä-Herttuala S. The syngeneic BT4C rat malignant glioma is a valuable model to study myelomonocytic cells in tumors. Cancer Growth Metastasis. 2012;5:CGM S9314 Available from: https://doi.org/10.4137/CGM.S9314.

30. Quail DF, Joyce JA. The microenvironmental landscape of brain tumors. Cancer Cell. 2017;31:326-41.

31. Morisse MC, Jouannet S, Dominguez-Villar M, Sanson M, Idbaih A. Interactions between tumor-associated macrophages and tumor cells in glioblastoma: unraveling promising targeted therapies. Expert Rev Neurother. 2018;18:729-37.

32. McCarthy JB, El-Ashry D, Turley EA. Hyaluronan, cancer-associated fibroblasts and the tumor microenvironment in malignant progression. Front cell Dev Biol. 2018:6:48.

33. Tammi Ml, Oikari S, Pasonen-Seppanen S, Rilla K, Auvinen P, Tammi RH. Activated hyaluronan metabolism in the tumor matrix - causes and consequences. Matrix Biol. 2019;78-79:147-64.

34. Mooney KL, Choy W, Sidhu S, Pelargos P, Bui TT, Voth B, et al. The role of CD44 in glioblastoma multiforme. J Clin Neurosci Off J Neurosurg Soc Australas. 2016;34:1-5.

35. Harvima IT, Naukkarinen A, Harvima RJ, Aalto ML, Neittaanmaki H, Horsmanheimo M. Quantitative enzyme-histochemical analysis of tryptaseand chymase-containing mast cells in psoriatic skin. Arch Dermatol Res. 1990;282:428-33.

36. Benarafa C, Simon H-U. Role of granule proteases in the life and death of neutrophils. Biochem Biophys Res Commun. 2017;482:473-81.

37. Bachovchin DA, Ji T, Li W, Simon GM, Blankman JL, Adibekian A, et al. Superfamily-wide portrait of serine hydrolase inhibition achieved by libraryversus-library screening. Proc Natl Acad Sci. 2010;107:20941 LP-20946 Available from: http://www.pnas.org/content/107/49/20941.abstract.

38. Swedberg JE, Li CY, de Veer SJ, Wang CK, Craik DJ. Design of potent and selective cathepsin $\mathrm{G}$ inhibitors based on the sunflower trypsin inhibitor-1 scaffold. J Med Chem. 2017;60:658-67 Available from: https://doi.org/10. 1021/acs.jmedchem.6b01509.

39. van Rooden EJ, Kreekel R, Hansen T, Janssen APA, van Esbroeck ACM, den Dulk $\mathrm{H}$, et al. Two-step activity-based protein profiling of diacylglycerol lipase. Org Biomol Chem. 2018;16:5250-3.

40. Dudani JS, Ibrahim M, Kirkpatrick J, Warren AD, Bhatia SN. Classification of prostate cancer using a protease activity nanosensor library. Proc Natl Acad Sci U S A. 2018;115:8954-9.

41. Shaul ME, Fridlender ZG. Cancer-related circulating and tumor-associated neutrophils - subtypes, sources and function. FEBS J. 2018;285:4316-42.

42. Massara M, Persico P, Bonavita O, Mollica Poeta V, Locati M, Simonelli M, et al. Neutrophils in Gliomas. Front Immunol. 2017:8:1349.

43. Jeon H-Y, Ham SW, Kim J-K, Jin X, Lee SY, Shin YJ, et al. Ly6G(+) inflammatory cells enable the conversion of cancer cells to cancer stem cells in an irradiated glioblastoma model. Cell Death Differ. 2019;26:2139.

44. Mulvihill MM, Benjamin DI, Ji X, Le Scolan E, Louie SM, Shieh A, et al. Metabolic profiling reveals PAFAH1B3 as a critical driver of breast cancer pathogenicity. Chem Biol. 2014;21:831-40.

45. Chang JW, Zuhl AM, Speers AE, Niessen S, Brown SJ, Mulvihill MM, et al. Selective inhibitor of platelet-activating factor acetylhydrolases $1 \mathrm{~b} 2$ and $1 \mathrm{~b} 3$ that impairs cancer cell survival. ACS Chem Biol. 2015;10:925-32.

46. Kohnz RA, Mulvihill MM, Chang JW, Hsu K-L, Sorrentino A, Cravatt BF, et al. Activity-based protein profiling of oncogene-driven changes in metabolism reveals broad dysregulation of PAFAH1B2 and 1B3 in cancer. ACS Chem Biol. 2015;10:1624-30

47. Viader A, Ogasawara D, Joslyn CM, Sanchez-Alavez M, Mori S, Nguyen W, et al. A chemical proteomic atlas of brain serine hydrolases identifies cell type-specific pathways regulating neuroinflammation. Elife. 2016;5:e12345. 
48. Edgington-mitchell LE, Barlow N, Aurelio L, Samha A, Szabo M, Graham B, et al. Fluorescent diphenylphosphonate-based probes for detection of serine protease activity during inflammation. Bioorg Med Chem Lett. 2017 27:254-60 Available from: https://doi.org/10.1016/j.bmcl.2016.11.064.

49. Tammi R, Agren UM, Tuhkanen AL, Tammi M. Hyaluronan metabolism in skin. Prog Histochem Cytochem. 1994:29:1-81.

50. Kuhns DB, Long Priel DA, Chu J, Zarember KA. Isolation and functional analysis of human neutrophils. Curr Protoc Immunol. 2015;111:7.23.1-7.23. 16.

51. Maqbool M, Vidyadaran S, George E, Ramasamy R. Optimisation of laboratory procedures for isolating human peripheral blood derived neutrophils. Med J Malaysia. 2011;66:296-9.

52. Kurkinen KM, Koistinaho J, Laitinen JT. [Gamma-35S] GTP autoradiography allows region-specific detection of muscarinic receptor-dependent Gprotein activation in the chick optic tectum. Brain Res. 1997;769:21-8.

53. Navia-Paldanius D, Savinainen JR, Laitinen JT. Biochemical and pharmacological characterization of human a/ $\beta$-hydrolase domain containing 6 (ABHD6) and 12 (ABHD12). J Lipid Res. 2012;53:2413-24.

\section{Publisher's Note}

Springer Nature remains neutral with regard to jurisdictional claims in published maps and institutional affiliations.

Ready to submit your research? Choose BMC and benefit from:

- fast, convenient online submission

- thorough peer review by experienced researchers in your field

- rapid publication on acceptance

- support for research data, including large and complex data types

- gold Open Access which fosters wider collaboration and increased citations

- maximum visibility for your research: over $100 \mathrm{M}$ website views per year

At BMC, research is always in progress.

Learn more biomedcentral.com/submissions 